\title{
The Post-periapsis Evolution of Galactic Center Source G1: The Second Case of a Resolved Tidal Interaction with a Supermassive Black Hole
}

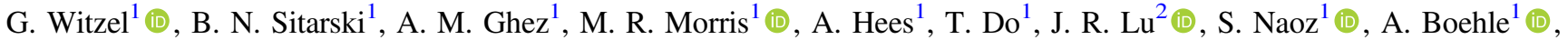 \\ G. Martinez ${ }^{1}$, S. Chappell ${ }^{1}$, R. Schödel ${ }^{3}$, L. Meyer ${ }^{1}$, S. Yelda ${ }^{1}$ (D), E. E. Becklin ${ }^{1,4}$, and K. Matthews ${ }^{5}$ \\ ${ }^{1}$ Department of Physics and Astronomy, University of California, Los Angeles, 430 Portola Plaza, Los Angeles, CA 90095-1547, USA; witzel@astro.ucla.edu \\ ${ }^{2}$ Institute for Astronomy, University of Hawaii, 2680 Woodlawn Drive, Honolulu, HI 96822, USA \\ ${ }^{3}$ Instituto de Astrofisica de Andalucia (CSIC), Glorieta de la Astronomia S/N, E-18008 Granada, Spain \\ ${ }^{4}$ NASA-Ames Research Center, MS 232-12, Moffett Field, CA 94035, USA \\ 5 Division of Physics, Mathematics, and Astronomy, California Institute of Technology, Pasadena, CA 91125, USA \\ Received 2015 November 25; revised 2017 July 15; accepted 2017 July 17; published 2017 September 22
}

\begin{abstract}
We present new adaptive optics (AO) imaging and spectroscopic measurements of Galactic center source G1 from W. M. Keck Observatory. Our goal is to understand its nature and relationship to G2, which is the first example of a spatially resolved object interacting with a supermassive black hole (SMBH). Both objects have been monitored with AO for the past decade (2003-2014) and are comparatively close to the black hole $\left(a_{\min } \sim 200-300\right.$ au) on very eccentric orbits $\left(e_{\mathrm{G} 1} \sim 0.99 ; e_{\mathrm{G} 2} \sim 0.96\right)$. While $\mathrm{G} 2$ has been tracked before and during periapsis passage $\left(T_{0} \sim 2014.2\right)$, G1 has been followed since soon after emerging from periapsis $\left(T_{0} \sim 2001.3\right)$. Our observations of G1 double the previously reported observational time baseline, which improves its orbital parameter determinations. G1's orbital trajectory appears to be in the same plane as that of G2 but with a significantly different argument of periapsis $\left(\Delta \omega=21^{\circ} \pm 4^{\circ}\right)$. This suggests that G1 is an independent object and not part of a gas stream containing G2, as has been proposed. Furthermore, we show for the first time that (1) G1 is extended in the epochs closest to periapsis along the direction of orbital motion, and (2) it becomes significantly smaller over time (450 au in 2004 to less than 170 au in 2009). Based on these observations, G1 appears to be the second example of an object tidally interacting with an SMBH. G1's existence $14 \mathrm{yr}$ after periapsis, along with its compactness in epochs further from the time of periapsis, suggest that this source is stellar in nature.
\end{abstract}

Key words: binaries: general - galaxies: star clusters: general - Galaxy: center - infrared: stars - quasars: supermassive black holes - stars: kinematics and dynamics

\section{Introduction}

As the capabilities of high-resolution imaging facilities have advanced, the center of our Galaxy has become a unique laboratory for studying the nearest supermassive black hole (SMBH; Ghez et al. 1998, 2008; Gillessen et al. 2009) and has revealed many unexpected results. This includes the presence of young stars where none were expected (e.g., Levin \& Beloborodov 2003; Genzel et al. 2003; Paumard et al. 2006; Bartko et al. 2009; Lu et al. 2009; Yelda et al. 2014), a lack of old stars where many were predicted (e.g., Buchholz et al. 2009; Do et al. 2009a), and very faint but highly variable infrared emission believed to be associated with the black hole's accretion flow.

The most recent Galactic center discovery from highresolution infrared observations that has attracted considerable attention is the very red infrared source G2, which recently went through closest approach, where its tidal interaction should have been maximal $\left(T_{0, \mathrm{G} 2}=2014.21 \pm 0.13\right.$; Meyer et al. 2014). It was originally hypothesized to be a 3 Earth-mass gas cloud, and as it went through closest approach to the SMBH Sgr A*, it was projected to tidally disrupt, shock, and possibly contribute to an enhanced accretion episode onto the black hole (Burkert et al. 2012; Gillessen et al. 2012; Schartmann et al. 2012; Pfuhl et al. 2015). Observations of G2 after periapsis passage have challenged the gas cloud hypothesis. First, it survived as a compact source in the continuum imaging measurements at $3.8 \mu \mathrm{m}$ (Witzel et al. 2014) and possibly as a compact source in the gas ( $\mathrm{Br} \gamma$ spectroscopic measurements; Valencia-S. et al. 2015). This has favored the alternative hypothesis that there is a central stellar source embedded in G2. There are several variants of the stellar hypothesis, including a disrupted protoplanetary disk (MurrayClay \& Loeb 2012), a disrupted disk around an old star (Miralda-Escudé 2012), a mass-loss envelope from a young T Tauri star (Scoville \& Burkert 2013), a Wolf-Rayet star (Eckart et al. 2013), spherically symmetric winds from an embedded object (Ballone et al. 2013), a binary merger product (Phifer et al. 2013; Witzel et al. 2014; Prodan et al. 2015), and an embedded pre-main-sequence star (Valencia-S. et al 2015).

More recently, another object $-\mathrm{G} 1$ - has been recognized as bearing a close relationship to G2. G1 was originally found to be another very red, extended infrared source that was interpreted as a spatially resolved, stationary hot dust feature that is locally heated by nearby stars surrounding $\mathrm{Sgr} \mathrm{A}^{*}$ (Clénet et al. 2004, 2005; Ghez et al. 2005b). In addition, Pfuhl et al. (2015) noted that G1 has observational properties similar to those of $\mathrm{G} 2$, including $\mathrm{Br} \gamma$ emission, as well as a very red color. Also, G1 passed through periapsis $\sim 13$ yr ago (Pfuhl et al. 2015; Sitarski et al. 2014), and therefore high-resolution observations only exist post-periapsis passage, while we have observations of G2 prior to, through, and post-periapsis passage. The observations in Pfuhl et al. (2015) also suggest that G1 moves on a Keplerian orbit with orbital characteristics similar to those of G2 (Sitarski et al. 2014). These similar orbits and observational characteristics led Pfuhl et al. (2015) to hypothesize that G2 and G1 are part of a gas streamer on the same trajectory. 
Table 1

Summary of Keck/NIRC2 L' $\left(\lambda_{0}=3.8 \mu \mathrm{m}\right)$ Data

\begin{tabular}{|c|c|c|c|c|c|c|c|c|c|c|c|}
\hline UT Date & $\begin{array}{l}\text { Decimal } \\
\text { Date }\end{array}$ & $t_{\text {int }} \times$ coadds & $\begin{array}{l}\text { Frames } \\
\text { Taken }\end{array}$ & $\begin{array}{l}\text { Frames } \\
\text { Used }\end{array}$ & Array Size & $\begin{array}{l}\text { Dithered } \\
\text { FOV }\end{array}$ & $\begin{array}{c}\text { PSF } \\
\text { FWHM } \\
\text { (mas) }\end{array}$ & Strehl & $\begin{array}{l}\mathrm{L}^{\prime}{ }_{\lim }^{\mathrm{a}} \\
(\mathrm{mag})\end{array}$ & $\begin{array}{c}\delta_{x} \\
(\mathrm{mas})\end{array}$ & $\begin{array}{c}\text { Original } \\
\text { Publication }^{\mathrm{b}}\end{array}$ \\
\hline 2002 May 31 & 2002.413 & $0.50 \times 40$ & 53 & 25 & $10^{\prime \prime} \times 10^{\prime \prime}$ & $11 " .6 \times 10 " .5$ & 100 & 0.26 & 12.0 & 2.2 & 0 \\
\hline 2003 Jun 10 & 2003.440 & $0.50 \times 40$ & 12 & 6 & $10^{\prime \prime} \times 10^{\prime \prime}$ & $12 ! .8 \times 10 " .2$ & 85 & 0.41 & 13.3 & 1.4 & 0 \\
\hline 2004 Jul 26 & 2004.567 & $0.25 \times 120$ & 11 & 11 & $10^{\prime \prime} \times 10^{\prime \prime}$ & $10 " .7 \times 9 ! .8$ & 80 & 0.42 & 14.4 & 0.4 & 1 \\
\hline $\begin{array}{c}2005 \mathrm{Jul} 30 \\
\text { and } 31\end{array}$ & 2005.580 & $0.50 \times 60$ & 62 & 56 & $10^{\prime \prime} \times 10^{\prime \prime}$ & $10 " .3 \times 9 " .5$ & 81 & 0.36 & 14.4 & 0.3 & 1 \\
\hline 2006 May 21 & 2006.385 & $0.50 \times 60$ & 19 & 19 & $10^{\prime \prime} \times 10^{\prime \prime}$ & $11 " .4 \times 11 " ! 3$ & 82 & 0.38 & 14.4 & 0.4 & 2 \\
\hline 2009 Jul 22 & 2009.556 & $0.50 \times 60$ & 4 & 4 & $10^{\prime \prime} \times 10^{\prime \prime}$ & $13 " ! 1 \times 12$ "! 3 & 85 & 0.38 & 13.2 & 0.1 & 3 \\
\hline 2012 Jul 20-23 & 2012.551 & $0.50 \times 30$ & 1316 & 1231 & $2 ! .64 \times 2 ! .64^{\mathrm{c}}$ & $2 ! .6 \times 2 . .8$ & 92 & 0.51 & 15.3 & 0.2 & 3 \\
\hline 2013 Aug 13 & 2013.616 & $0.50 \times 60$ & 249 & 245 & $2 ! .64 \times 2 ! .64^{c}$ & $2 ! .7 \times 2 ! .6$ & 90 & 0.54 & 15.2 & 0.1 & 4 \\
\hline 2014 Mar 20 & 2014.216 & $0.50 \times 60$ & 21 & 21 & $10^{\prime \prime} \times 10^{\prime \prime}$ & $10 " .3 \times 9 " .3$ & 91 & 0.51 & 14.4 & 0.4 & 5 \\
\hline 2014 May 11 & 2014.359 & $0.50 \times 60$ & 9 & 9 & $10^{\prime \prime} \times 10^{\prime \prime}$ & $10 " ! 1 \times 9 " .3$ & 90 & 0.53 & 13.2 & 0.4 & 5 \\
\hline 2014 Jul 2 & 2014.503 & $0.50 \times 60$ & 20 & 20 & $10^{\prime \prime} \times 10^{\prime \prime}$ & $10 " .2 \times 9 ! ! 3$ & 91 & 0.34 & 14.1 & 0.4 & 5 \\
\hline 2014 Aug 4 & 2014.590 & $0.50 \times 60$ & 28 & 28 & $10^{\prime \prime} \times 10^{\prime \prime}$ & $10 " .2 \times 9 " .4$ & 92 & 0.50 & 13.7 & 0.4 & 5 \\
\hline 2016 May 21 & 2016.376 & $0.50 \times 60$ & 25 & 25 & $10^{\prime \prime} \times 10^{\prime \prime}$ & $10 " .6 \times 9 " .3$ & 85 & 0.38 & 13.8 & 0.4 & 4 \\
\hline
\end{tabular}

Notes.

a This is defined as the $95 \%$ quantile of the distribution of magnitudes of detected stars with StarFinder.

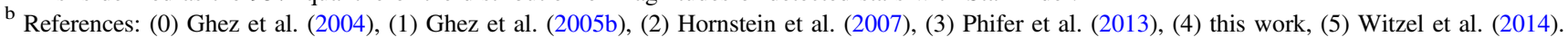

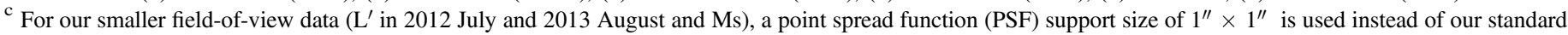

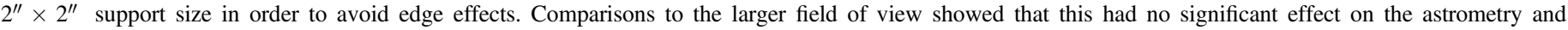
photometry of G1.

In this paper, we explore the evolution of G1's observed properties and orbital motion over the last decade, the longest time baseline reported thus far for this object. We investigate the evolution of G1 with time and position from $\mathrm{Sgr}^{*}$ to characterize its tidal interactions. With our longer time baseline, we test the theory that G1 and G2 are part of the same gas streamer.

This paper is organized as follows. Section 2 describes our data sets and data reduction techniques, Section 3 details our astrometric and photometric calibration and our orbital fitting procedure, Section 4 presents our results, and Section 5 discusses our findings in the context of G2 and evidence that these are self-gravitating objects. One scenario that we consider is the binary merger hypothesis. Section 6 summarizes our conclusions.

\section{Data Sets}

Near-infrared, high-angular-resolution images and spectra of the Galactic center region containing G1 have been obtained as part of the long-term program at the W. M. Keck Observatory (WMKO) carried out by our group to study the Galactic center black hole and its environs. In this paper, the primary data sets are WMKO images that have been acquired through the $\mathrm{L}^{\prime}$ $\left(\lambda_{0}=3.8 \mu \mathrm{m}\right)$ broadband filter over a $13 \mathrm{yr}$ period with NIRC2, the facility near-infrared camera (PI: K. Matthews) fed by the Keck II laser guide star adaptive optics system (LGSAO; van Dam et al. 2006; Wizinowich et al. 2006). Ten of the 12 epochs have been previously reported by us and are part of our group's archive of fully calibrated data sets (Ghez et al. 2004, 2005b; Hornstein et al. 2007; Phifer et al. 2013; Witzel et al. 2014). Two additional epochs of observation, 2013 August and 2016 May, are reported here for the first time. The pixel scale for these data sets is 9.950 mas pixel $^{-1}$ (Yelda et al. 2010), which corresponds to an oversampling factor of $\sim 9$ for a typical point-spread function. Table 1 summarizes all the $\mathrm{L}^{\prime}$ imaging data sets for this study.
The new $\mathrm{L}^{\prime}$ data sets were observed and calibrated using the same techniques described in the papers reporting our other $\mathrm{L}^{\prime}$ data sets (Stolte et al. 2010; Phifer et al. 2013; Witzel et al. 2014). This followed standard techniques with the exception of the treatment of the sky exposures, which were taken for each field rotator mirror position within the same range as the science data in steps of $\sim 2^{\circ}$. For each $L^{\prime}$ science exposure in epochs after 2004, the corresponding sky exposure was subtracted in order to accurately subtract the thermal emission from dust on the mirror optics (e.g., Stolte et al. 2010). Once the data were fully calibrated, selected frames were combined into an average map (main map). The individual frames were selected based on the image quality as measured by the full width at half maximum (FWHM) of the point spread function (PSF) $\left(\mathrm{FWHM} \leqslant 1.25 \times \mathrm{FWHM}_{\text {min }}\right.$, where $\mathrm{FWHM}_{\text {min }}$ is the minimum measured FWHM of all of the data) and were weighted by the Strehl ratio of each image. We additionally created three independent images (sub-maps) from three interleaved subsets of frames to determine astrometric and photometric uncertainties for the images.

For this study, we also draw upon two other types of imaging data sets. The first consists of two Ms $\left(\lambda_{0}=4.67 \mu \mathrm{m}\right)$ observations obtained on 2005 July 16 (previously published by Hornstein et al. 2007) and another obtained on 2016 May 21 . These were added to enhance our photometric characterization of G1. Second, we use all of our group's $\mathrm{K}^{\prime}$ data sets, which cover the central $10^{\prime \prime} \times 10^{\prime \prime}$ of our Galaxy and have been obtained to track the orbital motions of stars at the Galactic center (Ghez et al. 1998, 2000, 2003, 2005a, 2008; Lu et al. 2009; Yelda et al. 2010, 2014; Meyer et al. 2012; Boehle et al. 2016). In addition to the previously published $\mathrm{K}^{\prime}$ data sets, two new data sets, obtained on 2013 July 20 and 2016 May 21, are included in this work. The first data set was taken in an identical way to that of all previous $\mathrm{K}^{\prime}$ astrometric maps (e.g., Yelda et al. 2014) and consists of 193 frames of data, and its final combined image has a point-spread function with a 


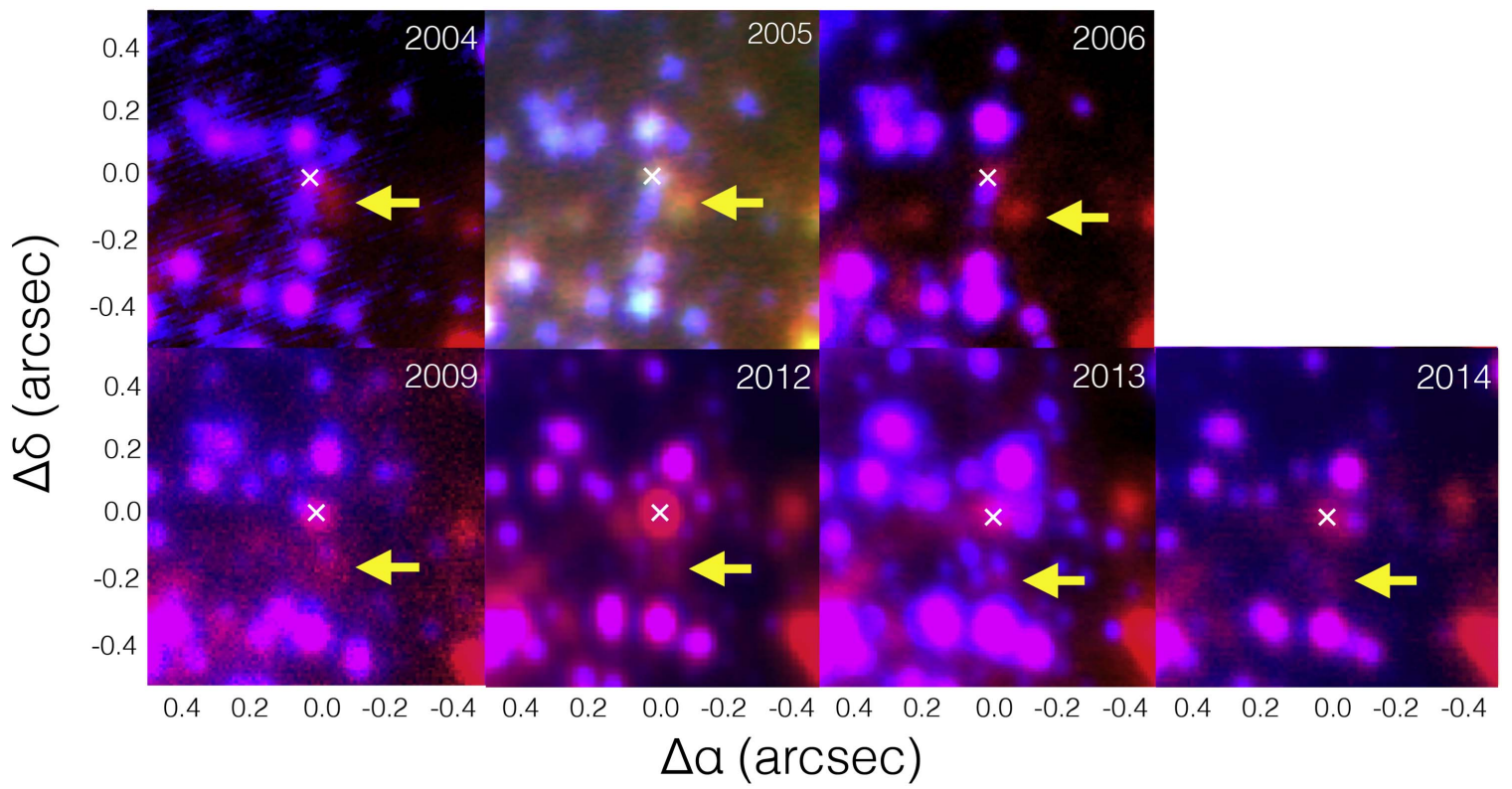

Figure 1. Two-color images made by combining NIRC2 images at $\mathrm{K}^{\prime}(2.2 \mu \mathrm{m}$, blue $)$ and $\mathrm{L}^{\prime}(3.8 \mu \mathrm{m}$, red). The images have been aligned using the coordinates of S0-2 from our respective StarFinder runs for each filter during each epoch; the 2014 data are from our 2014 March observation. The position of Sgr A* is denoted by the white " $\times$," and the position of G1 is denoted by a yellow arrow. The 2005 panel shows a three-color image that includes our $2005 \mathrm{Ms}$ data (2.2 $\mu \mathrm{m}$ in blue, $3.8 \mu \mathrm{m}$ in green, $4.7 \mu \mathrm{m}$ in red). G1 and G2 are distinctly red sources. Other red sources exist within the inner 0 " 5 of Sgr A* as well. It is apparent that Sgr A* varies considerably. For contour plots of G1, see Figure 6.

FWHM of 58.5 mas and a Strehl ratio of 0.36. The second was taken similarly to our $\mathrm{L}^{\prime}$ observations, in which we stared at a fixed position in the central field to minimize overheads. This map consists of 21 frames of data, and its final combined image has a PSF with a FWHM of 68 mas and a Strehl ratio of 0.26. The $\mathrm{K}^{\prime}$ data are used for both the photometric and astrometric characterization of G1.

Additionally, we utilize a spectroscopic data set obtained at WMKO with OSIRIS (Larkin et al. 2006). This data set, which was originally published in Ghez et al. (2008), consists of 28 frames taken on 2006 June 18 and 30 and 2006 July 01 through the narrowband $\mathrm{Kn} 3$ filter $\left(\lambda_{0}=2.166 \mu \mathrm{m}\right)$ with the 35 mas pixel scale. These observations have a spatial resolution at $\operatorname{Br} \gamma$ of 67 mas and a spectral resolution of $\sim 3600$. These OSIRIS data constitute some of our deepest $\mathrm{Kn} 3$ observations prior to $2012,{ }^{6}$ with a total integration time of $\sim 7 \mathrm{hr}$. While at that time, G1 was near Sgr A*, it was sufficiently separated ( $r=0.114 \pm 0$ ". 009) for the positions of Sgr $\mathrm{A}^{*}$ and G1 to be disentangled.

\section{Analysis}

\subsection{Imaging Analysis}

Our imaging analysis is divided into two parts: (i) astrometric analysis using the PSF fitting tool StarFinder and (ii) photometric and size calculations using a PSF convolved with a 2D elliptical Gaussian. Both measurements are described in detail below.

\subsubsection{Astrometry}

G1 is visually identified in every $\mathrm{L}^{\prime}$ and Ms image (see Figure 1). Its astrometric properties in every image were obtained using the PSF fitting program StarFinder (Diolaiti

\footnotetext{
6 OSIRIS was moved from Keck II to Keck I in 2012 January, and the grating was upgraded in 2013 January; there have been small reduction artifacts that affect the detection of faint emission-line objects in crowded fields.
}

et al. 2000) in a manner similar to what has been outlined in previous works (Yelda et al. 2014 and references therein). We initially ran StarFinder using a correlation threshold of 0.8 and 0.6 in the main image and subimages, respectively, to identify candidate sources in our images. This resulted in G1 detections in 2004, 2005, 2006, 2012, and 2013 in the $\mathrm{L}^{\prime}$ data. In 2003, 2009, and all $2014 \mathrm{~L}^{\prime}$ epochs and both the Ms data sets, a different approach was necessary to capture G1 due to poorer data quality, although G1 can be visually identified (Figure 1). We therefore altered the search criteria to seek a point source within a 3 pixel box centered at the point of the highest flux count, at the approximate position of G1 using a modified version of StarFinder that searches for additional sources at a lower correlation (Boehle et al. 2016). We did not use the 2016 $\mathrm{L}^{\prime}$ data for astrometry, as we used the orbital model from 2003-2014 to predict the position of G1 in the 2016 data (see our photometric analysis, described in Section 3.1.2). With this modified procedure, G1 was detected in all epochs.

While G1 is extended in the early epochs (Section 4.2), we still use the StarFinder astrometry that reliably determines G1's centroid, as the residuals in each epoch look symmetric. Twodimensional Gaussian fits convolved with a point-spread function to G1 yielded peak positions consistent with the positions extracted from StarFinder.

The point sources identified in each epoch are matched across all epochs and transformed to a common coordinate system in which $\mathrm{Sgr} \mathrm{A}^{*}$ is at rest (see Yelda et al. 2010; Phifer et al. 2013; Yelda et al. 2014; for the application of our distortion solution to $\mathrm{L}^{\prime}$-band data, see Appendix A). Specifically, each $\mathrm{L}^{\prime}$ list of stellar positions, or star list, is aligned to the $\mathrm{K}^{\prime}$ star list that is nearest in time with the translation, rotation, and affine first-order transformation that is independent in $x$ and $y$. The transformed G1 position is added to the $\mathrm{K}^{\prime}$ star list, and the $\mathrm{K}^{\prime}$ star lists from 1995 to 2014 are aligned as described in our earlier works (e.g., Ghez et al. 2008; Yelda et al. 2014) using measurements of infrared astrometric 
Table 2

Data and Observed Properties of G1

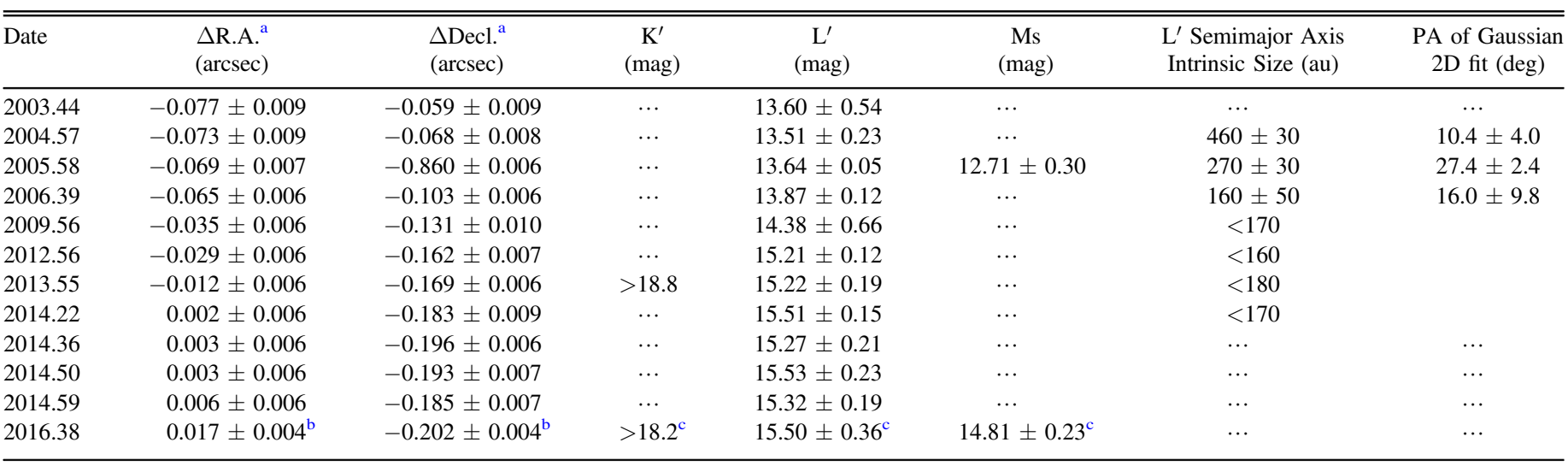

Notes.

a $\Delta$ R.A. and $\Delta$ Decl. are given in a reference frame in which the location of the black hole is not at the origin (see Table 4).

b These positions come from our orbital solution derived from our L' data taken from 2003 through 2014. See Section 3.3 for more information on our orbital fit.

${ }^{c}$ The photometry in 2016 was derived using deconvolved images rather than StarFinder, as was done in previous epochs. See Section 3.1.2 for more information.

secondary standards taken through 2012 (Yelda et al. 2010, 2014; Boehle et al. 2016). Table 2 lists the astrometry for $\mathrm{G} 1$ in each epoch prior to 2016.

\subsubsection{Photometry and Size Measurement}

The magnitudes of all point sources at $\mathrm{K}^{\prime}$ were calculated using PSF fitting with the StarFinder procedure (see previous section). We chose IRS 16C, IRS 16NW, and IRS 16CC as our photometric calibrators; this is part of our standard $\mathrm{K}^{\prime}$ calibration procedure (e.g., Yelda et al. 2014).

As G1 seems extended in 2004, 2005, and 2006 at $\mathrm{L}^{\prime}$ and in the $2005 \mathrm{Ms}$ data, we tested several photometric methods to obtain reliable photometry. To confirm whether the individual methods yielded reliable results during the epochs when G1 was visibly extended, we planted a 2D elliptical Gaussian model for the 2004 size (a Gaussian with a FWHM of 58 mas convolved with the PSF) in our data in three distinct regions: a high-background region, a low-background region, and near the position of $\mathrm{G} 1$. We planted sources with different brightnesses $\left(\operatorname{mag}_{\mathrm{L}^{\prime}}=10-16\right)$ to determine whether we could recover its magnitude and physical extent.

We tested three different photometric procedures: (1) StarFinder with a PSF support array of 2!"0 (200 pixels), following our standard $\mathrm{K}^{\prime}$ analysis; (2) StarFinder with a PSF support array of 0.9 (90 pixels) to make the PSF more robust against background artifacts at larger distances from the core; and (3) a 2D fit with an intrinsic extended elliptical Gaussian source convolved with an empirical PSF model. The planting simulations returned significantly decreased fluxes with respect to their original planted magnitude in the case of StarFinder PSF fitting with both PSF sizes (methods 1 and 2). However, method 3 reliably recovered the fluxes and observed extent of the planted sources to within $20 \%$ at the faintest magnitude tested $\left(\operatorname{mag}_{\mathrm{L}^{\prime}}=16\right)$.

We applied method 3 to every single $\mathrm{L}^{\prime}$ and Ms epoch (prior to 2016) using the IDL procedure mpfit2dfun. To prepare the images for model fitting, we first subtracted all $\mathrm{L}^{\prime}$-detected StarFinder sources. We then used the aligned $\mathrm{L}^{\prime}$ and $\mathrm{K}^{\prime}$ StarFinder star lists to find the position of $\mathrm{K}^{\prime}$-only detected sources and used the forced StarFinder algorithm from Boehle et al. (2016) to find the fluxes of these sources at $\mathrm{L}^{\prime}$. We subtracted these sources, as well as the StarFinder-generated backgrounds, from the original image. In our 2D elliptical Gaussian model, we allowed the position angle to vary and allowed for the FWHM to range between 0.3 and 10.0 pixels. If the FWHM of G1 fell below 0.3 pixels ( $\sim 3$ mas) in an epoch, then a PSF without a Gaussian component was used instead. Our photometry is reported in Table 2. A comparison of the astrometry between the three methods yielded identical positions of $\mathrm{G} 1$ within $1 \sigma$ errors.

To photometrically calibrate our $\mathrm{L}^{\prime}$ and Ms data, we used S0-2, S0-12, S1-20, and S1-1 and their L' magnitudes from Schödel et al. (2010). These sources were chosen because they are all in the field of view for every epoch, including our subarrayed epochs (see Table 1). Similarly to Schödel et al. (2011), the Ms data were calibrated using the same magnitudes as $\mathrm{L}^{\prime}$ because the relative colors of the calibrators are close to zero. The overall zero-point error from the Schödel et al. (2010) magnitudes is 0.15 mag at $\mathrm{L}^{\prime}$ and is not taken into account in Table 2 or Figure 2 because it affects all photometric measurements in the same way.

No $\mathrm{K}^{\prime}$ counterpart was detected for $\mathrm{G} 1$, and star-planting simulations were performed to determine an upper magnitude limit. We used the $\mathrm{L}^{\prime}$ position of G1 in the 2013 August image, where G1 is an isolated point source (see Figure 1) and transformed that into the 2013 July $\mathrm{K}^{\prime}$ coordinate system. The star-planting simulations were carried out using our modified version of StarFinder (Phifer et al. 2013; Boehle et al. 2016). There is a $\mathrm{K}^{\prime}$ source near G1 in 2013, S0-37, but it contributes at most $0.3 \mathrm{mJy}$ to the overall $\mathrm{L}^{\prime}$ flux of G1 (assuming the dereddening law outlined in Schödel et al. 2010 and that S0-37 has the same colors as S0-2). All photometry in each bandpass is reported in Table 2 .

The recovered sizes of G1 from our model show that G1 is extended between 2004 and 2006 but is consistent with a point source from 2009 through 2014 (see Table 2). The magnitudes and sizes of G1 as a function of time are shown in Figures 2 and 3, respectively, while Figure 4 shows the elongation of G1 in the direction of orbital motion in 2004. The major axis angle of the 2D elliptical Gaussian is consistent with a tangential line to the orbit in 2004 and 2005 (10.4 \pm 4.0 (tangent to orbit $=12.3 \pm 2.8^{\circ}$ ) and $27.4 \pm 4.8^{\circ}$ (tangent to orbit $=$ $21.0 \pm 2.4^{\circ}$ ) west of north, respectively; see Figure 4 ). 

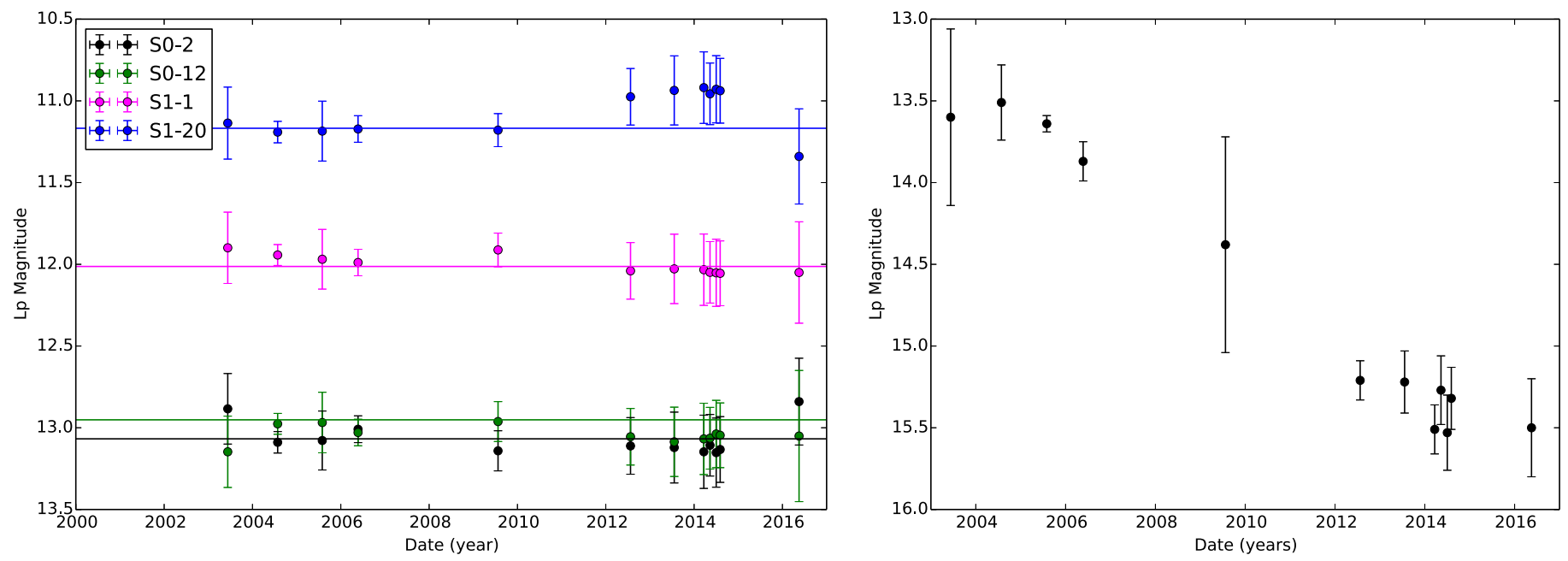

Figure 2. Left: L' photometry on each of our four calibration sources. The solid line denotes the reported magnitude from Schödel et al. (2011). Right: L' photometry of G1 as a function of time. The magnitude of the source has decreased significantly and varies directly with the size of the source presented in Figure 3 .

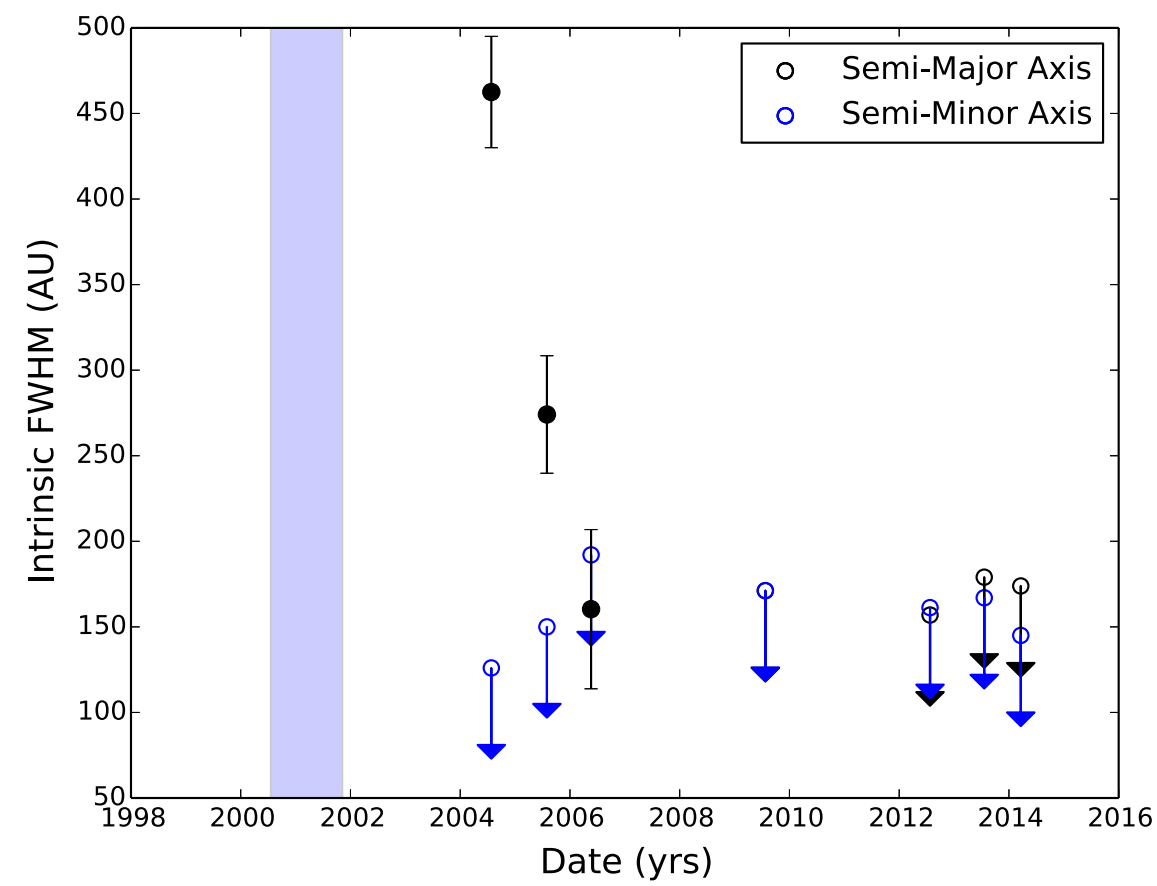

Figure 3. Size of G1 as a function of time since periapsis passage $\left(T_{0} \sim 2001.3\right)$ in both the semimajor and semiminor axis directions. In the epochs when G1 is resolved, we can get an actual measurement of the semimajor axis of the source; the last four epochs are upper limits on G1's size obtained by first subtracting the closest neighboring sources in that epoch (S0-2 and $\mathrm{Sgr} \mathrm{A}^{*}$ ) and then comparing the 2D Gaussian profile of G1 to the point-spread function. The blue bar denotes the FWHM of the 1D marginalized probability distribution function for the periapsis passage time.

In order to be able to infer the blackbody properties of $\mathrm{G} 1$ in a later epoch when the source is compact, we utilize $\mathrm{L}^{\prime}$ and $\mathrm{Ms}$ data from 2016. As G1 is in a confused region in this epoch, we adopt a different methodology to recover its $\mathrm{K}^{\prime}$ upper limit and $\mathrm{L}^{\prime}$ and Ms flux densities. The StarFinder-generated backgrounds are subtracted from each main map, and we subtract all StarFinder-identified point sources in the vicinity of our predicted position for G1. We then use the StarFinder-generated PSF to do an iterative Lucy-Richardson deconvolution (with 10,000 iterations). Aperture photometry is then performed after beam restoring at the predicted position of G1 based on its derived orbit from the 2003-2014 data. The photometry of the $2016 \mathrm{~L}^{\prime}$ data matches well with the 2014 epochs.

\subsection{Spectroscopic Analysis}

The radial velocity for G1 was obtained using a similar approach to that developed by Phifer et al. (2013) for G2: a spectrum of G1 was extracted from our 2006 OSIRIS data at a location that was determined by transforming the high-quality $2005 \mathrm{~L}^{\prime}$ star list to the OSIRIS coordinate system using a secondorder polynomial transformation. The spectrum was extracted using an aperture radius of 1 pixel (corresponding to 35 mas) and applying a local sky subtraction in an area clear of known contaminating stars (e.g., Do et al. 2013). The extracted spectrum was calibrated using the standard techniques (Do et al. 2009b), and the peak in the resulting spectrum was fit with a Gaussian model to derive an observed radial velocity and its FWHM. The 

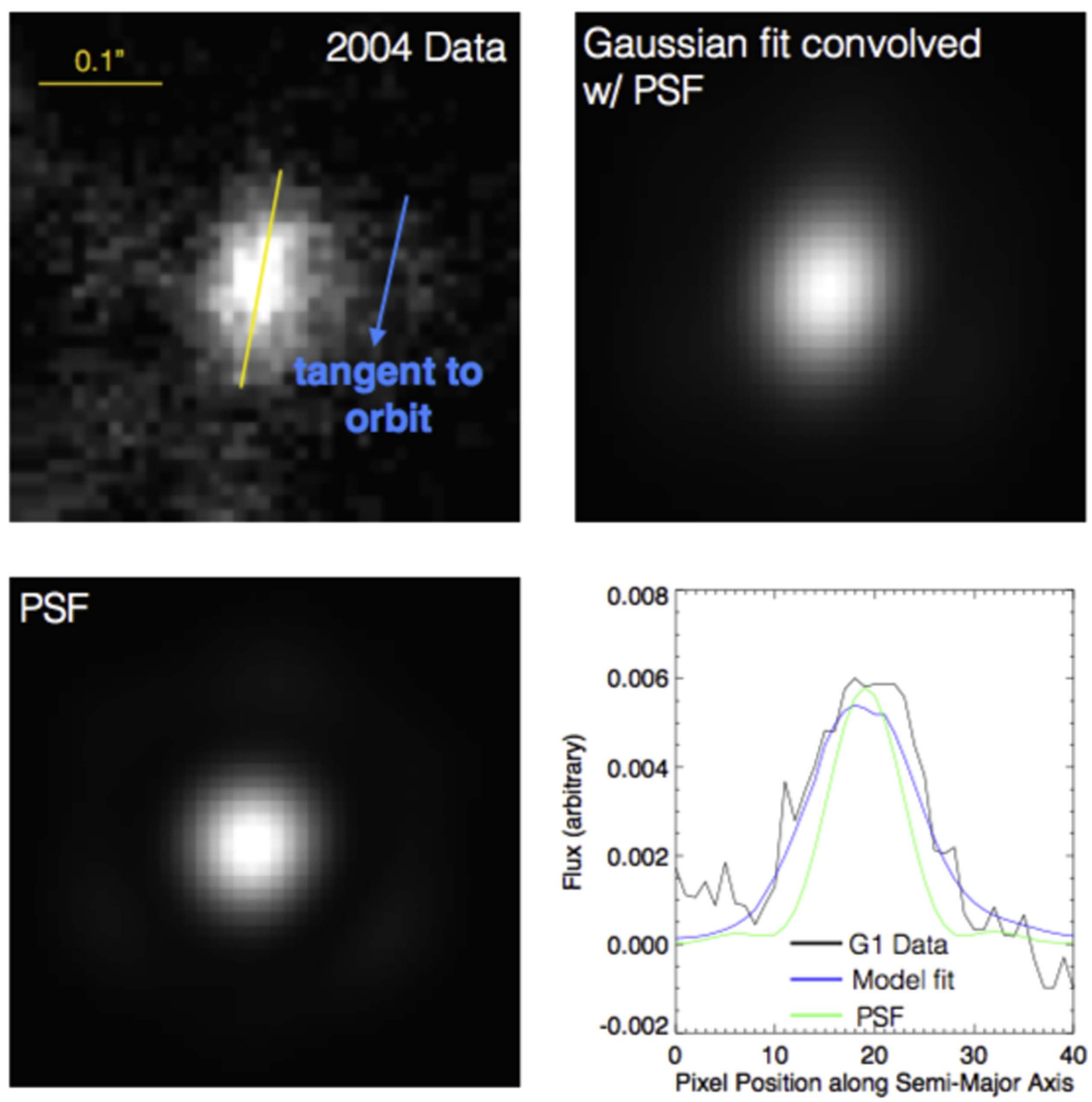

Figure 4. Upper left: G1 in 2004 after subtracting all L' StarFinder-detected point sources. The angle of the semimajor axis is denoted by the yellow line. The blue arrow shows a line in the direction of the tangent to the direction of orbital motion in 2004. Upper right: image of 2D Gaussian fit to the data found using mpfit2dpeak. Lower left: PSF model from 2004 as extracted from StarFinder. This panel and the upper left and upper right panels are all normalized so their peaks are on the same color table and scale; all panels are also on the same physical scale. Lower right: slice along the semimajor axis for our data (black line), the 2D Gaussian fit (blue line), and the PSF (green line). It is evident that the 2D Gaussian fit is an acceptable model for the $\mathrm{L}^{\prime}$ extension, and it is much larger than the PSF.

Table 3

Radial Velocity Data

\begin{tabular}{lccl}
\hline \hline Date & $\begin{array}{c}\text { Radial Velocity } \\
\mathrm{km} \mathrm{s}^{-1}\end{array}$ & $\begin{array}{c}\text { PSF FWHM } \\
\text { mas at Br } \gamma\end{array}$ & Orig. Publication \\
\hline 2004.6 & $-2043 \pm 150$ & & Pfuhl et al. (2015) \\
2006.2 & $-1594 \pm 163$ & & Pfuhl et al. (2015) \\
2006.5 & $-1558 \pm 60$ & 67 & This paper \\
2008.3 & $-1123 \pm 159$ & & Pfuhl et al. (2015)
\end{tabular}

resulting heliocentric radial velocity was corrected by $3.64 \mathrm{~km} \mathrm{~s}^{-1}$ to correspond to an local standard of rest (LSR) velocity of $-1568 \pm 60 \mathrm{~km} \mathrm{~s}^{-1}$ on the date of the observation (see Table 3). The FWHM of the spectral line is $185 \pm 41 \mathrm{~km} \mathrm{~s}^{-1}$. The spectrum and the corresponding point source in the line emission map are shown in Figure 5.

We compute the $\operatorname{Br} \gamma$ line luminosity similarly to Phifer et al. (2013) by comparing S0-2's flux density to G1's flux density. We estimate S0-2's dereddened flux density to be $14.1 \pm 0.2 \mathrm{mJy}$ (assuming the extinction prescription outlined in Schödel et al. 2010 and the flux density from Ghez et al. 2008) and compute an expected luminosity of S0-2 over the $2.15-2.159 \mu \mathrm{m}$ bandpass to be $0.16 L_{\odot}$. We then integrate over the same bandpass on the S0-2 and G1 spectra to get a final $\mathrm{Br} \gamma$ luminosity of G1 of (1.48 \pm $0.17) \times 10^{-3} L_{\odot}$. To check for consistency, we followed this same procedure to integrate over the same bandwidth for G2 (2.17-2.179 $\mu \mathrm{m})$, which yields a $\mathrm{Br} \gamma$ luminosity of $(1.36 \pm$ $0.25) \times 10^{-3} L_{\odot}$, consistent within $1 \sigma$ with the 2006 G2 luminosity reported in Phifer et al. (2013).

Using this dereddened $\mathrm{Br} \gamma$ line luminosity from 2006 (in an epoch where G1 is resolved), we can estimate the Ly $\alpha$ emission luminosity. We used the relationship between the $\mathrm{Br} \gamma$ emission and the free-free emission given in Wynn-Williams et al. (1978) and solved for the Ly $\alpha$ luminosity using the formulae summarized in Genzel et al. (1982) and Becklin et al. (1994). We estimate that the Ly $\alpha$ luminosity is $\sim 2 L_{\odot}$.

\subsection{The Orbital Determination of G1}

To derive the orbital properties of G1, we jointly fit for the Keplerian orbital parameters of S0-2, S0-38, and G1 (period, epoch of periapsis passage, eccentricity, position angle of the ascending node, argument of periapsis and inclination for each source) and the black hole parameters (the 2D position, the 3D velocity, and the mass of and distance to Sgr A*; see Table 4). S0-2 and S0-38 have complete orbital phase coverage and drive the black hole parameter fit. We use the same astrometry and 


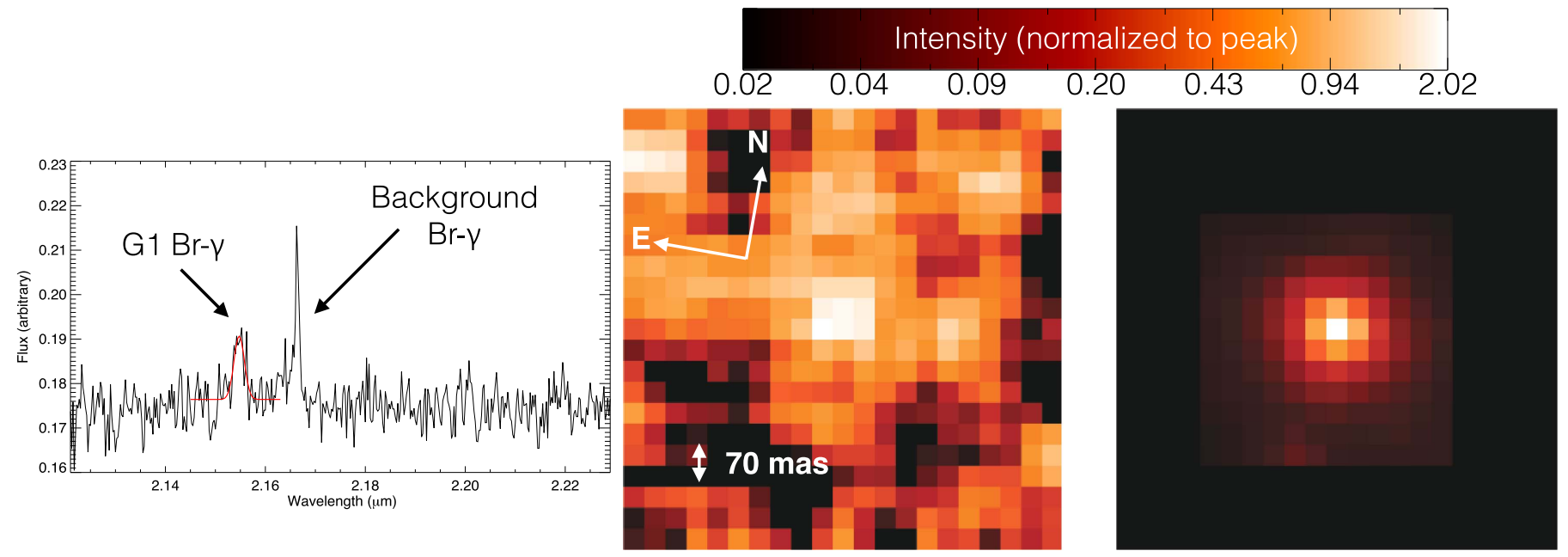

Figure 5. Left: continuum-subtracted spectrum of G1. The emission line at $2.154 \mu \mathrm{m}$ corresponds to a radial velocity of $-1568 \pm 60 \mathrm{~km} \mathrm{~s}{ }^{-1}$. This spectrum was extracted using an aperture of 1 pixel (35 mas) radius from our 2006 OSIRIS data cube. The overplotted red line shows the 1D Gaussian fit. Center: continuumsubtracted OSIRIS data cube collapsed over a $\Delta v$ of $267 \mathrm{~km} \mathrm{~s}^{-1}$ centered on $-1568 \mathrm{~km} \mathrm{~s}^{-1}$ (LSR-corrected) and smoothed over 2 spatial pixels. Right: Br $\gamma$ pointspread function extracted from our 2006 OSIRIS data cube. Both the PSF and the collapsed cube are displayed on the same physical scale and logarithmic color scale, where we normalize each figure to its respective peak.
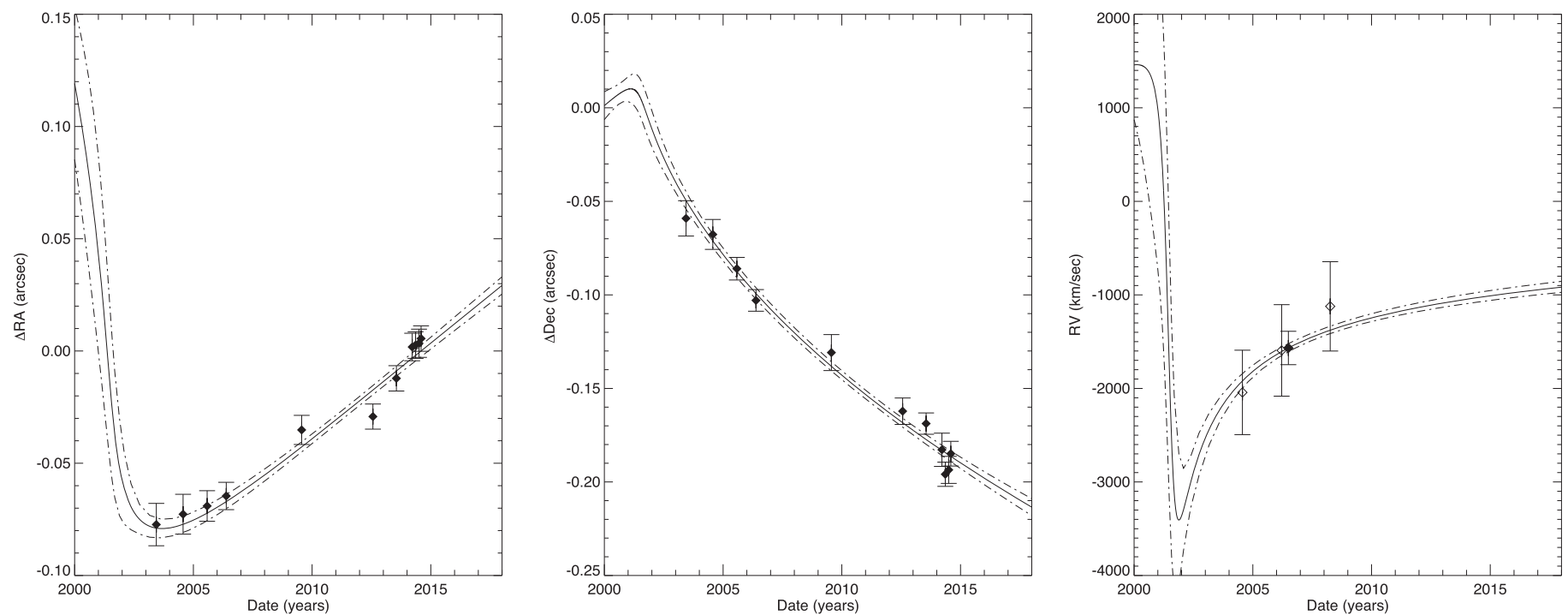

Figure 6. G1's kinematic measurements and best-fit orbital motion models. Our 11 astrometric measurements and one radial velocity measurement are shown as filled points. The three radial velocity (RV) measurements from Pfuhl et al. (2015) are plotted as open points. The $1 \sigma$ uncertainty envelopes are shown as dot-dashed lines.

radial velocities of S0-2 and S0-38 as reported in Boehle et al. (2016). Jointly fitting the three sources enables us to find an orbital solution for G1 and the black hole parameters. G1 alone does not have enough kinematic information to independently fit for the black hole parameters due to the lack of orbital phase coverage. We impose uniform, flat priors on each of the orbital parameters for $\mathrm{G} 1$ as follows: $\left[0^{\circ}, 360^{\circ}\right]$ for the argument of periapsis $(\omega),\left[0^{\circ}, 180^{\circ}\right]$ for the position angle of the ascending node $(\Omega),\left[0^{\circ}, 180^{\circ}\right]$ for the inclination, $[0,1]$ for the eccentricity, $[0,6000] \mathrm{yr}$ for the period, and $[1990,2010]$ for the time of periapsis passage. The G1 astrometry consists of 11 data points (see Section 3.1), including our newest astrometric measurements. For this orbital fit, we also used the three radial velocity measurements reported in Pfuhl et al. (2015) and our new measurement (Section 3.2; Table 3).

In this study, we include three sources of hypothetical systematics uncertainties: (i) source confusion, (ii) the presence of outliers, and (iii) uncertainty arising in the construction of the absolute reference frame.

Source confusion is a significant source of systematic error in our orbital fits, and the formal uncertainties are therefore underestimated. In order to account for this, we fit a secondorder polynomial to our astrometric data points and add a single additive value in quadrature to the formal errors until the final reduced $x^{2}$ of the second-order fit (that includes position, velocity, and acceleration) is equal to 1.0. The resulting additive value is 5.5 mas, which is roughly comparable to the formal uncertainties.

In order to assess the impact of hypothetical outliers, we use a jackknife resampling method, where we fit for G1's orbital parameters by dropping one epoch of observations at a time. This analysis, presented in Appendix B, shows results that are consistent with those reported in Table 4, and we conclude that no outlier significantly impacts our results. 
Table 4

S0-2 and S0-38 Black Hole Parameter Values

\begin{tabular}{lc}
\hline \hline Orbital Parameter & Peak Fit $^{\mathrm{a}}$ \\
\hline$X$-position of Sgr A* $\left(x_{0}\right.$, mas $)$ & $2.1_{-0.3}^{+0.5} \pm 1.90$ \\
$Y$-position of Sgr A* $\left(y_{0}\right.$, mas $)$ & $-4.4 \pm 0.4 \pm 1.23$ \\
$\Delta$ R.A. velocity of $\operatorname{Sgr~A}^{*}\left(V_{x}\right.$, mas yr $\left.^{-1}\right)$ & $-0.12_{-0.02}^{+0.03} \pm 0.13$ \\
$\Delta$ Decl. velocity of $\operatorname{Sgr~A}^{*}\left(V_{y}, \mathrm{mas} \mathrm{yr}^{-1}\right)$ & $0.68 \pm 0.05 \pm 0.22$ \\
Radial velocity of Sgr A $\left(V_{z}, \mathrm{~km} \mathrm{~s}^{-1}\right)$ & $-20.4 \pm 6.3 \pm 4.28$ \\
Distance to Sgr A $\left(R_{0}, \mathrm{kpc}^{-1}\right.$ & $7.87 \pm 0.11$ \\
Mass of Sgr A* $\left(M\right.$, millions of $\left.M_{\odot}\right)$ & $3.93_{-0.13}^{+0.07}$ \\
\hline
\end{tabular}

S0-2 Parameters

Time of closest approach $\left(T_{0}, \mathrm{yr}\right)$

Eccentricity $(e)$

Period $(P, \mathrm{yr})$

Angle to periapsis $(\omega, \mathrm{deg})$

Inclination $(i, \mathrm{deg})$

Position angle of the ascending node $(\Omega, \mathrm{deg})$

$2002.346 \pm 0.003$

$0.892_{-0.001}^{+0.002}$

$15.93_{-0.05}^{+0.02}$

$66.8_{-0.5}^{+0.3}$

$134.3 \pm 0.3$

$228.0_{-0.5}^{+0.4}$

S0-38 Parameters

Time of closest approach $\left(T_{0}, \mathrm{yr}\right)$

Eccentricity $(e)$

Period $(P, \mathrm{yr})$

Angle to periapsis $(\omega, \mathrm{deg})$

Inclination $(i, \mathrm{deg})$

Position angle of the ascending node $(\Omega, \mathrm{deg})$

$$
\begin{gathered}
2003.191_{-0.017}^{+0.038} \\
0.811 \pm 0.003 \\
19.22_{-0.2}^{+0.1} \\
13_{-21}^{+15} \\
169 \pm 2 \\
94_{-14}^{+18}
\end{gathered}
$$

Note.

${ }^{\mathrm{a}}$ The peak and corresponding $1 \sigma$ errors are from the marginalized 1D distributions for the respective parameters. The first error term corresponds to the error determined by the orbital fit itself, while the second error term on the black hole parameters refers to uncertainty in the reference frame and was determined by Boehle et al. (2016).

Following the procedure described in the Appendix of Boehle et al. (2016), the systematic uncertainties due to the construction of the absolute reference frame have been assessed by using a jackknife resampling method. In this jackknife analysis, one of the seven masers used to construct the reference frame is dropped at a time. Boehle et al. (2016) showed that this systematic uncertainty is important for the SMBH position and velocity relative to the origin of the constructed reference frame but is negligible for all other fitted parameters, such as the SMBH mass and distance $R_{0}$ and the orbital parameters. We conclude that systematic effects arising from the construction of our absolute reference frame do not significantly impact the posterior probability distribution for stellar orbital parameters.

\section{Results}

Our analysis of both photometric and spectroscopic information and our Keplerian orbital fit have led to three key results: G1 follows a highly eccentric Keplerian orbit that differs from G2's orbit; shortly after periapsis G1's L' emission is extended along the direction of orbital motion; and G1's L' emission is much larger than the tidal radius of even a $100 M_{\odot}$ source shortly after periapsis indicating that this emission comes from material that is not gravitationally bound to $\mathrm{G} 1$.

\subsection{Keplerian Orbital Fit Results}

The orbit of G1 is consistent with Keplerian motion (see Figures 7 and 8). Based on our precise astrometry and radial velocity points, G1 lies on a highly eccentric orbit $(e=$ $\left.0.99_{-0.01}^{+0.001}\right)$ and has recently passed through periapsis $\left(T_{0}=2001.3 \pm 0.4\right)$. The three orbital angles (position angle of the ascending node $\Omega=87.1_{-4.9}^{+5.0}$, argument of periapsis $\omega=117.3_{-2.9}^{+2.8}$, and inclination $i=109.0_{-0.8}^{+0.9}$ ) are well constrained, but the orbital period is very poorly constrained due to a lack of orbital phase coverage. The $1 \mathrm{D}$ and $2 \mathrm{D}$ joint posterior distribution functions are shown in Figures 9 and 10. The bestfit orbit is shown in Figure 8, and the peak of the 1D marginalized probability distribution functions along with the maximum likelihood best fit are presented in Table 5. We only fit bound, closed orbits; G1 could be on a hyperbolic orbit, since the eccentricity distribution is artificially truncated. The period restriction of $P<6000 \mathrm{yr}$ also constrains our orbital fits.

Figure 6 shows our orbital plots, extracted G1 astrometry, and radial velocity measurements. Figure 8 presents our projection of the orbit onto the sky and compares it to the orbital solution from Pfuhl et al. (2015) while assuming the black hole parameters from Gillessen et al. (2009). The left panel of Figure 8 clearly shows that our optimal G1 solution is significantly different from the previously published solution of Pfuhl et al. (2015). As a consequence, the best-fit G1 orbit is no longer in agreement with G2's optimal solution (right panel of Figure 8; G2's orbit is thoroughly discussed in Gillessen et al. 2012, 2013b, Phifer et al. 2013, and Meyer et al. 2014). This can be understood because our data cover almost twice the time baseline presented in Pfuhl et al. (2015).

\subsection{Size Variation}

In the epochs closest to periapsis G1 is extended along the direction of orbital motion (the semimajor axis). Figure 3 shows the intrinsic extent of G1 corrected for the size of the PSF along both the semimajor and semiminor axes. The source shape is approximately elliptic, and the semimajor axis of an elliptical 2D Gaussian fit is aligned with the direction of linear motion (Figure 4). However, in the more recent epochs, G1 becomes more compact. Additionally, there is significant brightness variation of $\mathrm{G} 1$ at $\mathrm{L}^{\prime}$ post-periapsis passage, which corresponds to its size evolution: when G1 is at its largest size, it is also brightest; when G1 is compact, it is $\sim 2$ mag dimmer. The arrows in Figure 3 show the intrinsic (PSF-size-corrected) upper limits on the source size along the semimajor and semiminor axes, which is, on average, $\sim 170$ au along the semimajor axis assuming $R_{0}=8 \mathrm{kpc}$.

Figure 7 shows images of G1 with all of the neighboring point sources identified by StarFinder subtracted. The contours illustrate the size development of G1. The FWHM of the semimajor axis of G1 is as high as $463 \pm 16$ au in 2004.567 after correcting for the PSF contribution (see Figure 3) but decreases to the size of a point source after 2006. Figure 11 shows azimuthally averaged radial profiles of G1 from 2009 through 2014, showing that the size of G1 is indeed consistent with a point source.

Our $2006 \mathrm{Br} \gamma$ detection is quite shallow, and we are unable to determine whether $\mathrm{G} 1$ is resolved at $\mathrm{Br} \gamma$. Due to the shallowness of the $\mathrm{Br} \gamma$ detection, we are unable to conclude if G1 is spatially resolved or has a velocity gradient. 

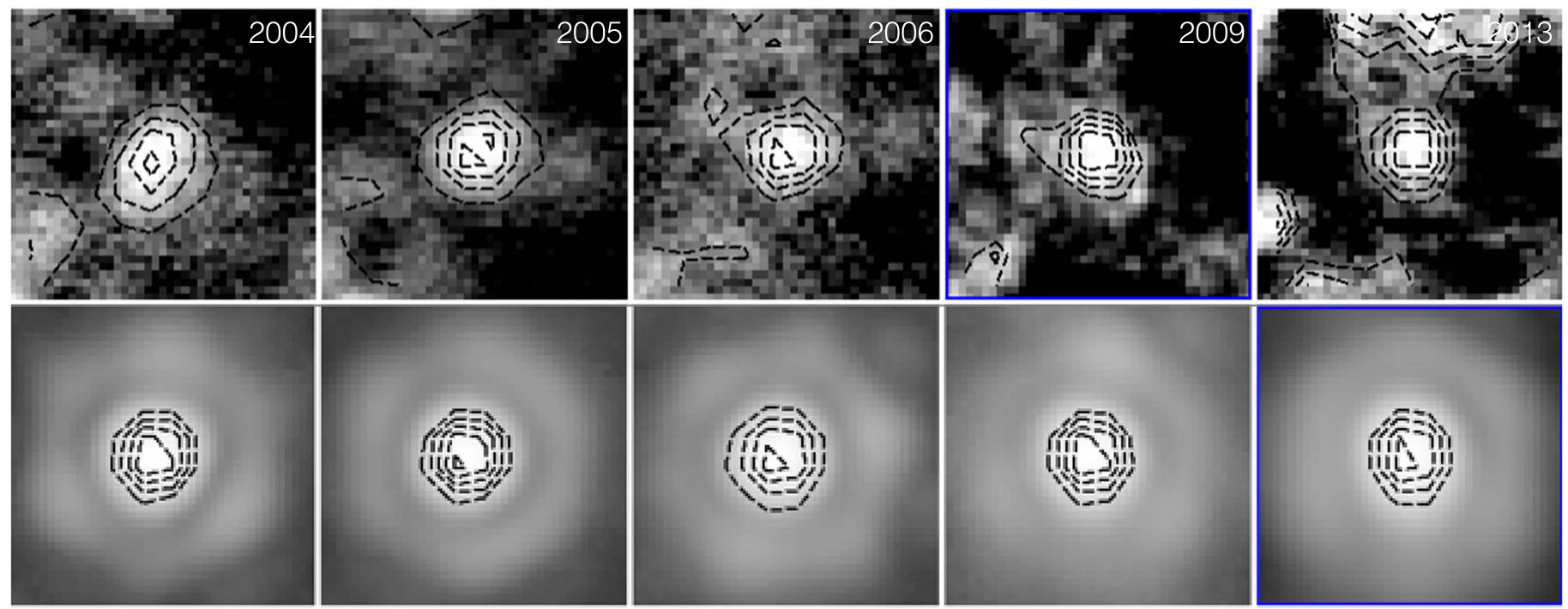

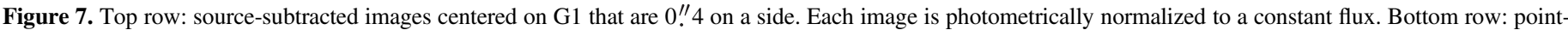

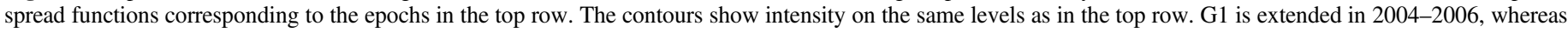
G1 is compact after 2009.

\subsection{Photometry and Temperature of G1}

There is a large photometric difference $(\sim 2 \mathrm{mag})$ between the epochs when G1 is extended (2004, 2005, and 2006) and when it is pointlike. The brightness develops with size, as epochs when G1 is extended are brightest and epochs when G1 is pointlike are dimmer and remain at a constant magnitude from 2012 through 2016.

G1 is identified at $\mathrm{L}^{\prime}$ and $\mathrm{Ms}\left(\mathrm{L}^{\prime}=13.65\right.$ in 2005; $\mathrm{Ms}=12.71$ in 2005) but not at $\mathrm{K}^{\prime}\left(\mathrm{K}^{\prime}>18.8\right.$ in 2013). Assuming zero-point fluxes for $\mathrm{L}^{\prime}$ from Tokunaga (2000) and the extinction law outlined in Schödel et al. (2010), we infer a dereddened $\mathrm{L}^{\prime}$ flux of $2.7 \pm 0.5 \mathrm{mJy}$ in 2005 .

In order to infer a temperature for G1 at a moment in time (2005) when it is extended enough to be resolved, we expect it to be optically thin, and therefore we use a modified blackbody,

$$
I_{\nu} \propto Q_{0}\left(\frac{\nu}{\nu_{0}}\right)^{\beta} B_{\nu}\left(T_{\mathrm{dust}}\right)
$$

where $\nu_{0}$ is the frequency at which the temperature is calculated, $Q_{0} / \nu_{0}^{\beta}$ is a constant, and $B_{\nu}$ is the Planck function. We take the power-law index $\beta$ equal to 2 , as in Lau et al. (2013) and consistent with extinction curves from Draine (2003). We separately do the same calculation assuming $\beta=0$ (blackbody). The temperature is therefore calculated following the equation

$$
\mathrm{L}^{\prime}-\mathrm{Ms}=-2.5 \log \left[\left(\frac{\nu_{\mathrm{L}^{\prime}}}{\nu_{\mathrm{Ms}}}\right)^{\beta} \frac{B_{\mathrm{L}^{\prime}}\left(T_{\text {dust }}\right)}{B_{\mathrm{Ms}}\left(T_{\text {dust }}\right)}\right] .
$$

From our $\mathrm{L}^{\prime}$ and Ms measurements, we are able to obtain a dereddened color $\left(\mathrm{L}^{\prime}-\mathrm{Ms}\right)$ of 0.706 . Fitting a modified blackbody following Equation (2) with $\beta=2$, the color temperature we obtain from our 2005 data is equal to $568 \pm 44 \mathrm{~K}$; assuming a blackbody $(\beta=0)$, we obtain a 2005 temperature of $426 \pm 44 \mathrm{~K}$, where our error bars are computed via a Monte Carlo simulation.
Using our $\mathrm{L}^{\prime}$ and Ms photometric data in 2016 when G1 is observed to be pointlike and assuming that G1 behaves as a blackbody in this epoch $(\beta=0)$, we infer a blackbody temperature of $684 \pm 75 \mathrm{~K}$ (where our error bars are again computed via a Monte Carlo simulation). Therefore, our inferred blackbody temperature has increased from 2005 to 2016 .

\section{Discussion}

G1 is a cold, extended source that has tidally interacted with $\mathrm{Sgr} \mathrm{A}^{*}$ and survived at least $13 \mathrm{yr}$ past periapsis passage. It has observable parameters that seem to be consistent with other examples of infrared excess sources at the Galactic center, the most prominent of which is G2. Many of its orbital and observable properties are comparable with those of G2: its cold temperature ( $426 \mathrm{~K}$ if $\beta=0$ or $568 \mathrm{~K}$ if $\beta=2$ in 2005 ; $684 \pm 75 \mathrm{~K}$ if $\beta=0$ in 2016), its highly eccentric orbit $\left(e=0.99_{-0.01}^{+0.001}\right)$, and the orientation of the orbital plane (see Table 5). There is a measurable size change post-periapsis passage, and the $\mathrm{L}^{\prime}$ flux density also changes dramatically after periapsis. In the following, we discuss the similarities and differences between $\mathrm{G} 1$ and $\mathrm{G} 2$.

\subsection{Is G1 Part of a Gas Streamer Common with G2?}

Pfuhl et al. (2015) recently proposed that G1 and G2 are not only lying in the same orbital plane but follow the same trajectory. They speculate that the Keplerian orbits of G1 and G2 are closely related, and they postulate that the small deviations between the orbits of the two objects are due to the drag force from the ambient Galactic center medium. This additional drag force leads to an evolution of G2's orbit into G1's orbit over time. Similarly, McCourt \& Madigan (2016) and Madigan et al. (2017) used G1 and G2 as probes to constrain the properties of the accretion flow surrounding Sgr $\mathrm{A}^{*}$. They modeled the orbital differences (as found by Pfuhl et al. 2015) between G1 and G2 in terms of an interaction with the background flow (McCourt \& Madigan 2016) and in the accretion flow onto Sgr A* (Madigan et al. 2017). Based on 

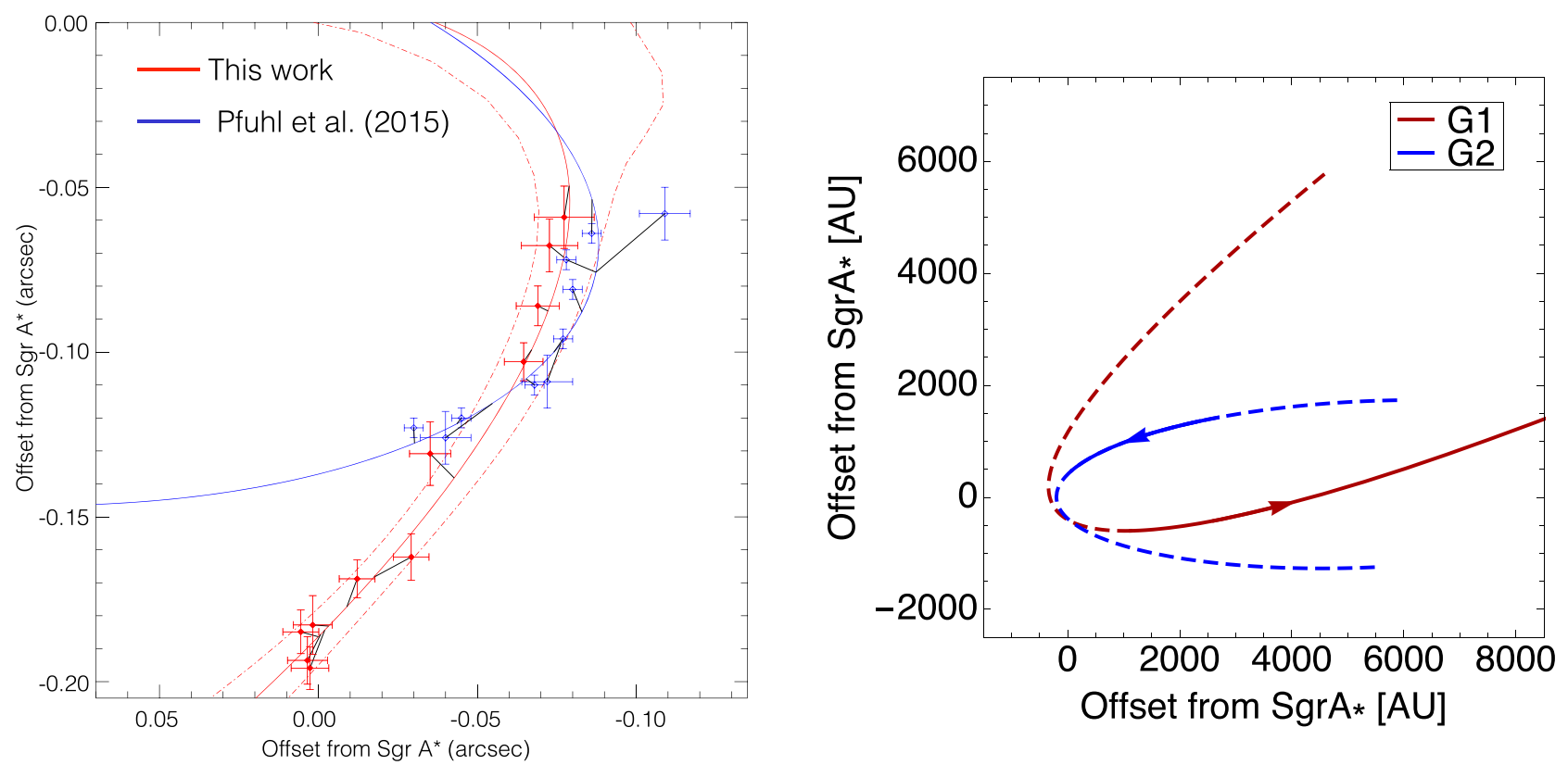

Figure 8. Left: comparison of G1's orbital solution in this work and Pfuhl et al. (2015). Our Keplerian orbital fit is shown in red, while the orbital fit and data from Pfuhl et al. (2015) are shown in blue. The black lines connect the observed point to the same point in time on the model orbit. There is an astrometric bias in 2009 and 2010 from confusion or a background dust emission feature that may skew the astrometry in those epochs. We do not use the astrometry from Pfuhl et al. (2015) due to differing reference frames. Right: orbits of G1 and G2 (as described in Table 5) projected into their common average orbital plane $\left(\Omega_{\mathrm{G} 1}=+2.5, \Omega_{\mathrm{G} 2}=-2.5^{\circ}\right.$, $i_{\mathrm{G} 1}=-2^{\circ}, i_{\mathrm{G} 2}=2^{\circ}, \omega_{\mathrm{G} 1}=117^{\circ}, \omega_{\mathrm{G} 2}=96^{\circ}$ ). It is evident that despite having similar orbital planes, the orbital trajectories are different. The solid (dashed) lines show times when we have (have not) observed G1 and G2.

their orbital analysis, they concluded that both sources could have originated from the clockwise young stellar disk (Paumard et al. 2006; Lu et al. 2009; Yelda et al. 2014).

However, the study we present here, which includes data taken several years beyond the last data point used in Pfuhl et al. (2015) (2014.6 versus 2010.5; true anomalies of $10.5^{\circ}$ and 8.7 , respectively), shows that despite the common orbital plane, G1 and G2 have distinct Keplerian orbits with a significant $(>3 \sigma)$ difference in their arguments of periapsis $\sim 3$ times larger than the difference reported in Pfuhl et al. (2015). This is demonstrated in Figure 7 showing both the data and the best-fit orbits projected into the plane of the sky, as well as both best-fit orbits projected into the average orbital plane.

Our findings do not firmly exclude the models proposed by McCourt \& Madigan (2016) and Madigan et al. (2017). However, while both models might be able to accommodate such a large change of the Keplerian orbit in the case of a compact gas cloud, the drag force scenario and a resulting common trajectory of G1 and G2 become increasingly unlikely in the context of a central star and thus larger object masses, as indicated by the compactness and brightness of both sources. The masses derived in the following sections and in Witzel et al. (2014) are $10^{5}-10^{6}$ times larger than the originally proposed 3 Earth masses. The interpretation in Pfuhl et al. (2015) that G1 and G2 are two dense regions within the same extended gas streamer that fills one trajectory around the black hole and have an identical origin but are offset by $\sim 13 \mathrm{yr}$ therefore seems unlikely.

The orbital planes of G1 and G2 are very similar, and they are fairly close to the plane defined by the clockwise disk (Yelda et al. 2014, and see Figure 10 of Pfuhl et al. 2015). G1 and G2 may have therefore originated the clockwise disk. We note, however, that there are other G2-like sources that do not lie on their common orbital plane (Sitarski et al. 2015).

\subsection{Evolution of G1's Dust Envelope}

Independent of whether G1 and G2 are related by a gas streamer, their physical natures are still not yet known. Recent results (e.g., Witzel et al. 2014; Valencia-S. et al. 2015, in contrast to Pfuhl et al. 2015) support the hypothesis that G2 has a stellar component due to its periapsis passage survival. This raises the question of whether there is similar evidence that G1 is stellar in nature.

In contrast to observations that $\mathrm{G} 2$ is unresolved at $\mathrm{L}^{\prime}$, for $\mathrm{G} 1$ we are able to measure its size in 2005 , and we can therefore put constraints on the optical depth, $\tau$, of the dust envelope at this point in time. Based on several parameters calculated in Section $4.3\left(T_{\beta=2}=568 \mathrm{~K}, T_{\beta=0}=426 \mathrm{~K}, r_{\mathrm{G} 1,2005}=137 \mathrm{au}\right)$, we find that the optical depth of G1 is small in the epochs when it is resolved, and we can therefore conclude that the origin of the extended continuum emission is an optically thin medium in 2005. As calculated in Section 3.2, the ambient radiation field in the Galactic center is strong enough (with Ly $\alpha$ alone) to externally heat this optically thin shell. The profile of G1 in the epochs where it is extended is well constrained by a PSF convolved with a 2D Gaussian (see Section 3.1.2) and shows no evidence of two components (as could be modeled by a PSF + a 2D Gaussian). This indicates that we do not see a central, optically thick point source in 2005.

From 2009 onward, G1 is unresolved at $\mathrm{L}^{\prime}$ and shows a significantly lower, roughly constant flux density of $\sim 0.6 \mathrm{mJy}$. Blackbody modeling of G1's L'-Ms color yields a temperature of $684 \mathrm{~K}$, implying a blackbody radius of $\sim 1$ au and a luminosity of $\sim 4.5 L_{\odot}$. This high luminosity and the fact that the object becomes more compact with time point to a substantially larger mass than 3 Earth masses. As indicated by the evolutionary tracks of main-sequence stars, this mass can be of the order of $1 M_{\odot}$ (Figure 12). However, the large derived blackbody size for the unresolved G1 shows that it is not a 


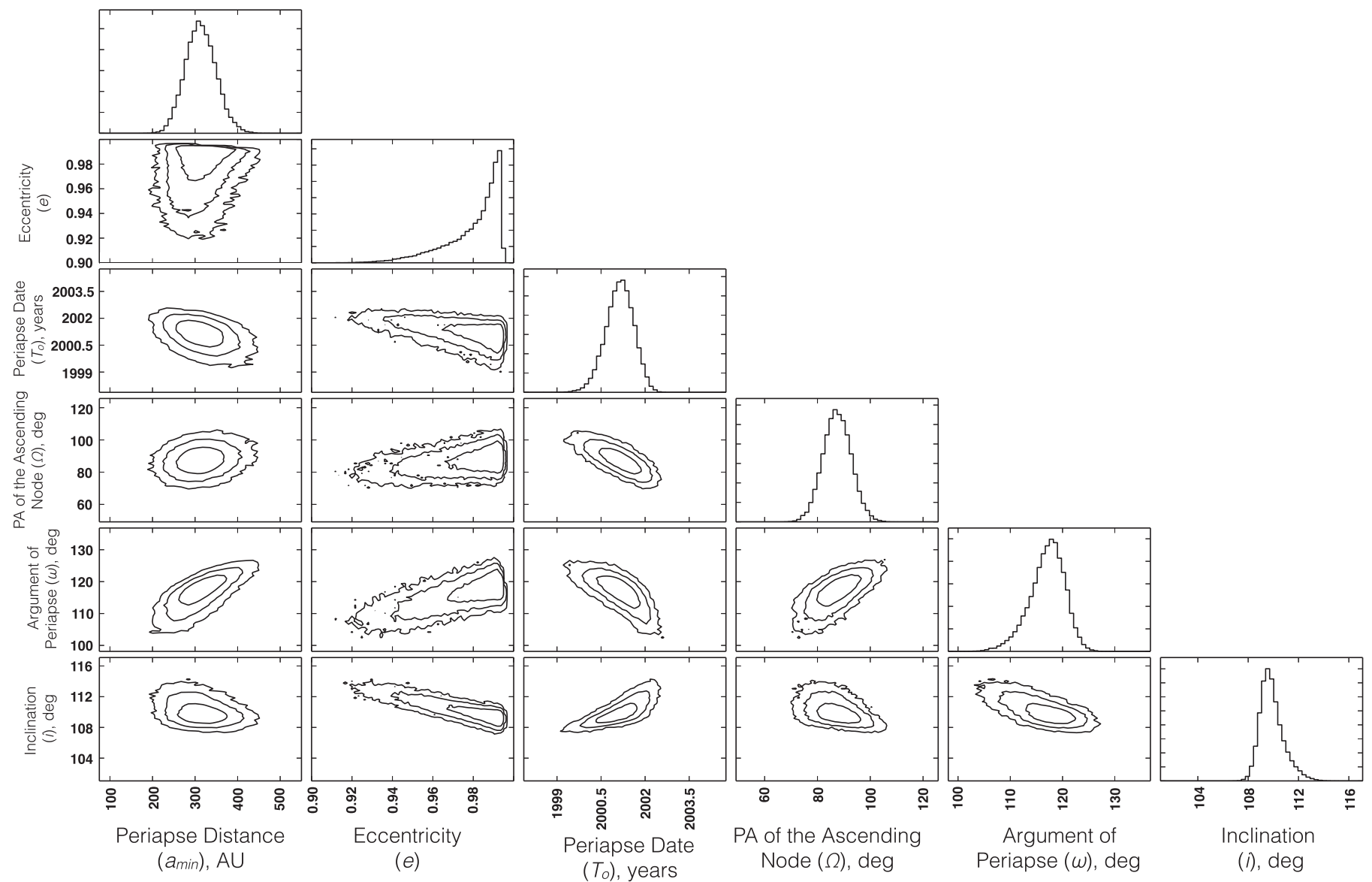

Figure 9. One-dimensional marginalized probability distribution functions for the six Keplerian orbital parameters for G1 ( $1 \sigma, 2 \sigma$, and $3 \sigma$ contours), along with the joint probability distribution functions for all parameters.

main-sequence star, nor is G1 luminous enough to be a red giant.

The material at the enormous radial distance from the center of G1 of $r \sim 230$ au of the outer halo seen in the extended epochs certainly remains unbound from G1 for even much higher G1 masses than $1 M_{\odot}$; in fact, this holds true for a central mass that is two orders of magnitude higher due to the weak $M^{1 / 3}$ dependence of the tidal radius. (Figure 13 shows the tidal radius (black lines) of a $2 M_{\odot}$ source (solid line) and a $100 M_{\odot}$ source (dashed line; see Witzel et al. 2014) plotted with the measured HWHMs of $\mathrm{G}^{*}$.) Therefore, this material is stripped, and its emission falls below the detection limit as its density decreases or grains are destroyed by $\mathrm{X}$-rays and highenergy particles generated in the accretion flow (e.g., Lau et al. 2015 and references therein; Tielens et al. 1994). It is interesting to note that the minimal radius of material that remains bound throughout periapsis for a G1 mass of $1 M_{\odot}$ and a periapsis passage distance of $\sim 300$ au is 1 au (see Figure 14, which plots the tidal radius as a function of time since periapsis passage for G1 and G2). This corresponds nicely to the derived blackbody radius in 2016 .

The question remains how G1 has reached the enormous extent of $d=460$ au in 2004. This likely requires that G1 was already large at periapsis passage. From an energy argument, we can determine the size of G1 at periapsis passage from the maximum shearing velocity, $v_{\mathrm{sh}}$, of the object in the potential of the black hole according to the equation

$$
\begin{aligned}
v_{\mathrm{sh}}^{2} & =\left(\frac{r_{\mathrm{obs}}-r_{\mathrm{per}}}{\Delta t}\right)^{2} \\
& =\left[\sqrt{v_{*}^{2}-2 G M_{\mathrm{BH}}\left[\frac{1}{d_{*}}-\frac{1}{d_{*}-r_{\mathrm{per}}}\right]}-v_{*}\right]^{2}-\frac{2 G m}{r_{\mathrm{per}}},
\end{aligned}
$$

where $r_{\text {obs }}$ is the observed size in 2004; $r_{\text {per }}$ is the half width along the Sgr $\mathrm{A}^{*}$-G1 line; $v_{*}$ is the velocity of G1 at periapsis passage; $m$ is the mass of $\mathrm{G} 1 ; M_{\mathrm{BH}}$ is the mass of the $\mathrm{SMBH}$ from Table $5 ; \Delta t$ is the difference between our observation date (2004.6) and periapsis passage time, the first epoch where we see a resolved $\mathrm{G} 1$; and $d_{*}$ is the distance from the center of $\mathrm{G} 1$ to Sgr A* Simultaneously solving for $m$ and $r_{\text {per, }}$, we find that $r_{\text {per }}$ is larger than 21 au at the time of closest approach and that the solution is not strongly mass dependent.

Therefore, for G1 to have its measured size be so large in $2004, r_{\text {per }}$ must be $\geqslant 21.3$ au at periapsis passage, and G1 was likely a large object even before it started interacting with the SMBH. If G1 began with a radius of 4,3 , or 2 au, it would have started interacting tidally with the SMBH $1.3,0.9$, or $0.4 \mathrm{yr}$ before periapsis passage, respectively, giving it plenty of time to grow to be the large source we infer for periapsis passage. 

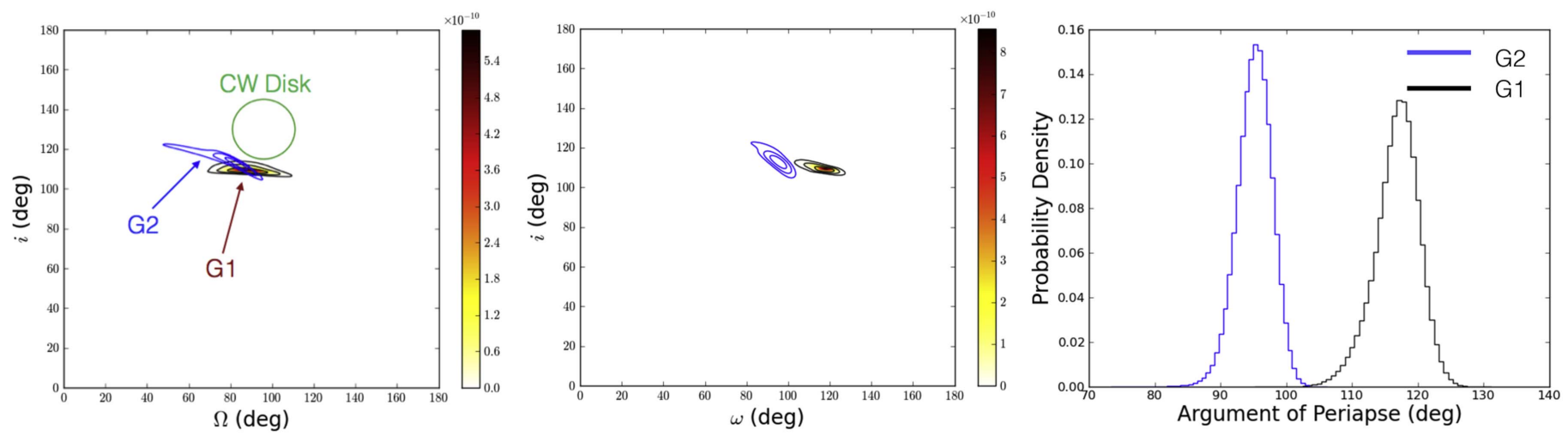

Figure 10. Joint probability distribution functions showing G1 (black) and G2 (blue) $3 \sigma$ contours. While G1 and G2 may have similar orbital orientations, as shown in the $\Omega$ vs. $i$ plot (left), their arguments of periapsis $(\omega)$ differ by greater than $3 \sigma$, implying that they have different orbits (center and right). The clockwise disk's orientation and width are overplotted on the $\Omega$ vs. $i$ plot to show the orientation of the orbital plane's proximity to the clockwise-moving disk of young stars.

Table 5

Orbital Parameters for G1 and G2

\begin{tabular}{|c|c|c|c|c|c|}
\hline Parameter & Best Fit, G1 & Peak, G1 ${ }^{\mathrm{a}}$ & Best Fit, G2 ${ }^{\mathrm{b}}$ & Peak, G2 ${ }^{\mathrm{b}}$ & $\begin{array}{c}\text { G1 Fit } \\
\text { Pfuhl et al. (2015) }\end{array}$ \\
\hline Time of closest approach $\left(T_{0}, \mathrm{yr}\right)$ & 2001.0 & $2001.3_{-0.2}^{+0.4}$ & 2014.1 & $2014.2_{-0.05}^{+0.03}$ & $2001.6 \pm 0.1$ \\
\hline Eccentricity $(e)$ & 0.981 & $0.992_{-0.01}^{+0.002}$ & 0.962 & $0.964_{-0.073}^{+0.036}$ & $0.860 \pm 0.050$ \\
\hline Argument of periapsis $(\omega, \mathrm{deg})$ & 118 & $117 \pm 3$ & 95 & $96 \pm 2$ & $109 \pm 8$ \\
\hline Inclination $(i, \mathrm{deg})$ & 109 & $109 \pm 1$ & 112 & $113 \pm 2$ & $108 \pm 2$ \\
\hline Position angle of the ascending node $(\Omega, \mathrm{deg})$ & 89 & $88_{-4}^{+5}$ & 83 & $82 \pm 2$ & $69 \pm 5$ \\
\hline
\end{tabular}

Notes.

${ }^{\mathrm{a}}$ The errors reported here are the $1 \sigma$ errors taken from the marginalized 1D distributions for the respective parameters.

${ }^{\mathrm{b}}$ G2 parameters are from performing an orbital fit on our available astrometric and spectroscopic points (those outlined in Meyer et al. 2014) in the fashion described in Section 3.3.

The clockwise disk parameters are $i=130^{\circ} \pm 15^{\circ}$ and $\Omega=96^{\circ} \pm 15^{\circ}$, where $15^{\circ}$ reflects the half width at half maximum (HWHM) from the peak density of the clockwise disk, as reported in Yelda et al. (2014).

While it is possible that $\mathrm{G} 1$ appears extended at $\mathrm{L}^{\prime}$ because of confusion with background sources, we judge this to be unlikely. We have traced the orbits of all known stars close to G1 and Sgr A*, and G1 is certainly not confused with a bright $\left(\operatorname{mag}_{\mathrm{L}^{\prime}}<16\right)$ source. But it is not fully excluded that, during the early epochs, there could be several dim stars whose images are overlapping that of nearby G1 for multiple epochs before separating and moving below the detection limit again. However, the symmetry in the extended residual after subtracting a point source makes this seem rather unlikely.

\subsection{The "Bloated Star" Model}

Our suggested model for G1's dust shell is as follows. G1 started tidally interacting with the SMBH with a rather large size several years prior to periapsis passage. The tidal radius penetrated deep into the dust shell $(r \sim 1 \mathrm{au})$, and the outer part of the optically thick shell became unbound from the source. This unbound shell became optically thin and externally heated by the surrounding radiation field in the Galactic center, which is what we observe starting in 2004 . Over time, the tidally stripped dust expanded away and fell below the detection limit against the local background emission, and, by 2009, we see the optically thick surface of a massive, internally heated object as a point source that is $2 \mathrm{mag}$ fainter than what is observed in 2004. Throughout all epochs, the source is also surrounded by an externally heated gas envelope that we observe as $\mathrm{Br} \gamma$ emission. As we discuss in Section 5.5, a possible physical explanation for G1's large size is that it could be an example of a black-hole-driven binary merger product (Phifer et al. 2013; Witzel et al. 2014; Prodan et al. 2015).

\subsection{Comparison to G2: Gas Cloud or Star?}

Several predictions have been made for the post-periapsis development of G2 in the case of a pure gas cloud. G1 and G2 have similar periapsis passage distances and blackbody sizes (as inferred in Section 4.3 for G1), and we expect them to tidally interact with the black hole in a comparable manner. Thus, in the following, we compare G1's post-periapsis observables to some of these predictions for G2.

Various models for G2 predict that if it were a pure gas cloud, it should undergo tidal shearing within 1 to $7 \mathrm{yr}$ after periapsis. The $\mathrm{Br} \gamma$ flux of $\mathrm{G} 2$ was predicted to rapidly decrease over time (Anninos et al. 2012; Morsony et al. 2015), due to both the breakup of $\mathrm{G} 2$ and the heating of its gas. Observationally, the latest $\mathrm{Br} \gamma$ line detection of G1 occurred in 2008, 7 yr after periapsis passage (Pfuhl et al. 2015), ${ }^{7}$ not showing any indication of a strong decay or complete

\footnotetext{
7 In epochs later than 2008, the $\operatorname{Br} \gamma$ line is extremely difficult to extract due to the insufficient quality of our data in these epochs.
} 

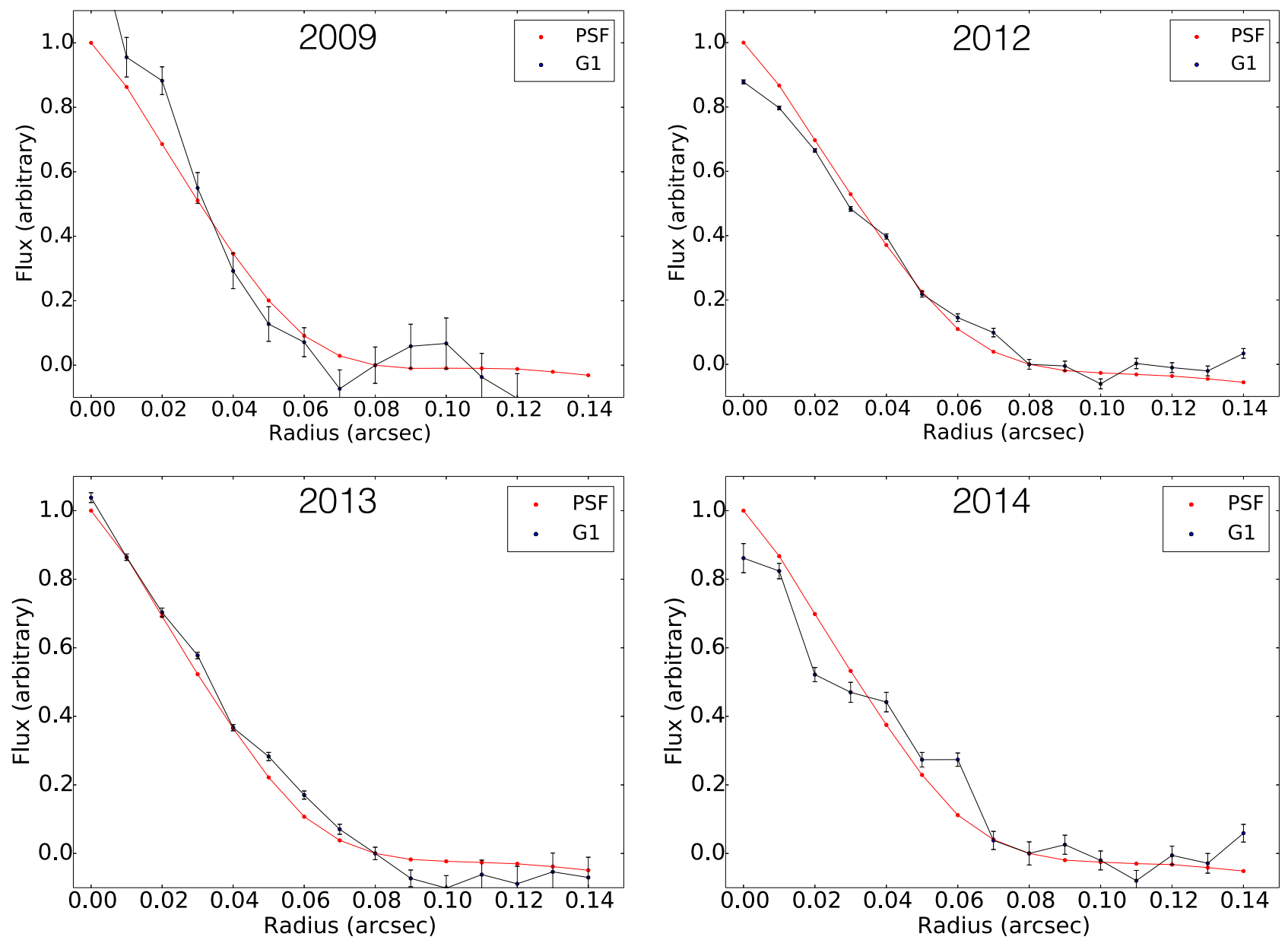

Figure 11. Azimuthally averaged radial profiles for G1 from 2009 through 2014. It is evident that the size of G1 becomes consistent with our StarFinder-extracted model of our point-spread function.

depletion. In fact, the post-periapsis luminosity of G1 is consistent with the pre-periapsis luminosity of G2 (see Section 3.2). We also note that G1's FWHM in 2006, $5 \mathrm{yr}$ after periapsis passage, was $185 \mathrm{~km} \mathrm{~s}^{-1}$, comparable to the line width of G2 $5 \mathrm{yr}$ before its periapsis passage in 2014 (Phifer et al. 2013). This provides strong constraints on future hydrodynamic modeling of the post-periapsis development of these objects.

Unlike G2, whose flux density stayed constant before and during periapsis (Witzel et al. 2014), G1's L' flux significantly decreased post-periapsis (Figure 2). The size of G1 at L' shows a similar decrease over time from a resolved, optically thin source $2 \mathrm{yr}$ after periapsis to an unresolved, compact source $5 \mathrm{yr}$ post-periapsis passage. Our calculation in the previous section indicates that $\mathrm{G} 1$ went through periapsis passage with a radius $>21$ au. These findings suggest that G1's smaller mass and larger size caused its dust envelope to have a stronger tidal interaction with $\operatorname{Sgr} A^{*}$ than that of G2. While they have similar tidal radii close to periapsis passage, G1 interacts with the SMBH for a longer period of time than $\mathrm{G} 2$.

Several studies (e.g., Anninos et al. 2012; Gillessen et al. 2012, 2013a; Schartmann et al. 2012; Morsony et al. 2015) find that if $\mathrm{G} 1$ or $\mathrm{G} 2$ were a gas cloud, there should be a significant increase in the steady-state X-ray flux several months before and after periapsis passage due to shocks. The Chandra X-ray
Observatory was launched in 1999, and the earliest observations of $\mathrm{Sgr} \mathrm{A}^{*}$ were conducted in late 1999 and 2000. Baganoff et al. (2001) and Ponti et al. (2015) showed no indication of an increase in the steady-state X-ray flux in the time around G1's periapsis passage (2001.3 \pm 0.4 ; Figure 3 in Ponti et al. 2015), although they raised the possibility that there was an increase in the rate of bright flares.

The size evolution of G1 in $\mathrm{L}^{\prime}$; the fact that both sources are on distinct Keplerian orbits (which disfavors their origin in a common gas stream), as described in Section 5.1; the intact $\mathrm{Br} \gamma$ emission after $7 \mathrm{yr}$; the survival of $\mathrm{G} 1$ at $\mathrm{L}^{\prime}$; and the lack of an increased X-ray flux all support the hypothesis that G1 has a massive $\left(\sim 1 M_{\odot}\right)$ central (stellar) component surrounded by an envelope of gas and dust, similar to our hypothesis for G2 (Witzel et al. 2014). Even if the mass of G1 is smaller than $1 M_{\odot}$, it is still $\sim 10^{5}$ times larger than the masses suggested for a gas cloud (Gillessen et al. 2012; Pfuhl et al. 2015).

\subsection{Comparison to Observed Merged Binary Systems}

G1 shares some observed characteristics with known merged binaries. In this section, we qualitatively examine the plausibility of the hypothesis that G1 and G2 are binary merger products. Our inferred dust temperature of 430-570 K is within the ranges reported for other observed binary mergers, 


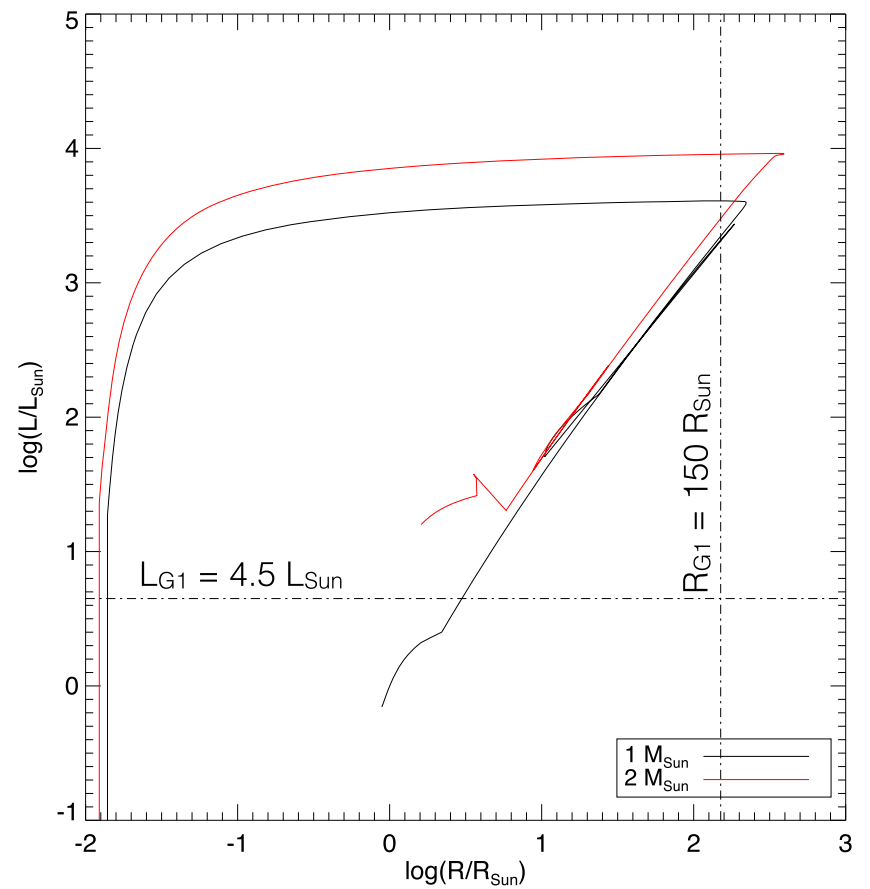

Figure 12. Evolutionary tracks of stars of 1 (black) and 2 (red) $M_{\odot}$ in the luminosity-radius plane, computed using the single-star evolution (SSE) code (Hurley et al. 2000). The vertical and horizontal lines show the inferred blackbody values for G1 from our 2016 data set. It is clear that the luminosity we infer for G1 is too small for a source we see of that radius.

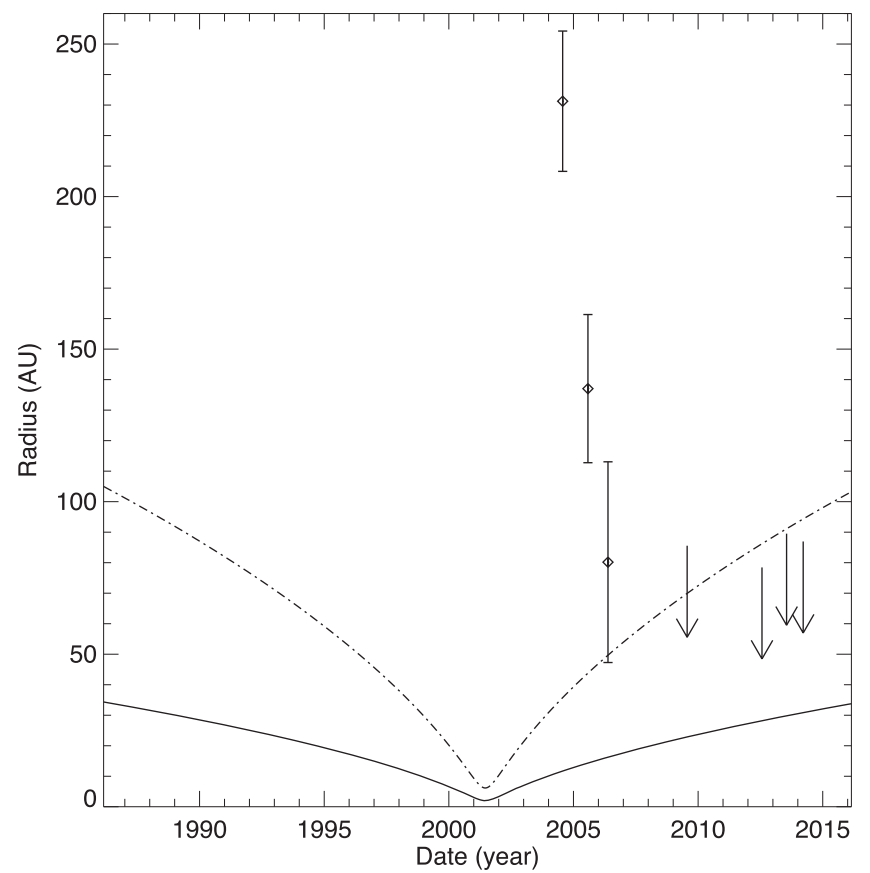

Figure 13. Tidal radius as a function of time since periapsis passage for G1 The solid line shows the tidal radius of a $2 M_{\odot}$ main-sequence star (as found for G2 in Witzel et al. 2014); the dot-dashed line shows the tidal radius of a $100 M_{\odot}$ star. The intrinsic size of the semimajor axes of G1 from Figure 3 are overplotted as well. It is evident that in the epochs where G1 is large, it lies well outside the tidal radius and therefore must interact gravitationally with Sgr $\mathrm{A}^{*}$. Therefore, some of the dust has become unbound. The remainder found in later epochs survives as a compact object.

including V1309 Sco (Nicholls et al. 2013) and BLG-360 (Tylenda et al. 2013). The large size and luminosity inferred for G1 are similar to those for BLG-360. The high infrared flux

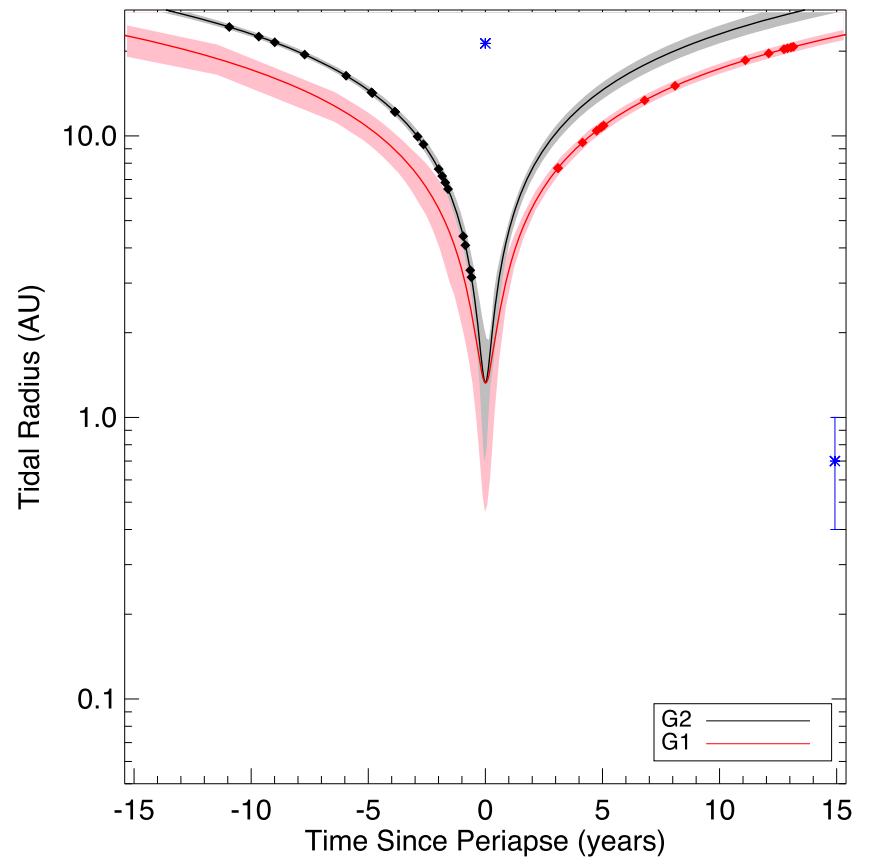

Figure 14. Plot of the tidal radii of G1 (red) and G2 (black). The tidal radius for G1 is computed assuming a mass of $1.0 M_{\odot}$, consistent with our luminosity calculation described in Section 5.2. The first blue asterisk denotes the inferred size of G1 at periapsis passage calculated with our dynamical model; the second blue asterisk shows the inferred size assuming that G1 is a blackbody in 2016. The latter is consistent with $1.0 \mathrm{au}$, the deepest point of direct tidal interaction of G1 with Sgr $\mathrm{A}^{*}$. G1 has a longer interaction with Sgr $A^{*}$ than G2 does.

density of G1 (2.7 mJy at $\left.\mathrm{L}^{\prime}\right)$ shortly after periapsis passage (2005) is consistent with the high fluxes from other binary merger products after the merger has taken place. However, while binary mergers show similarities to the observed characteristics of G1, many aspects regarding merger processes and products remain unclear. Observationally, we know very little about the lifetime of merger products. We can only provide observational evidence for lower limits of a few years. For instance, V1309 Sco was originally discovered in 2008 September as a "red nova" (Nakano et al. 2008; Rudy et al. 2008a, 2008b; Tylenda et al. 2011) that had an evolving spectral type from F to M. Nicholls et al. (2013) showed that V1309 Sco was undetected in the mid-infrared regime prior to its outburst; $\sim 23$ months afterward, the authors found a clear near- and mid-infrared excess. In 2012, 4 yr after its outburst, the infrared excess was still present (Tylenda \& Kamiński 2016), stemming from a dust envelope reaching as far as a few thousand au from the object. Interpreting the G-sources as merger products would require that the duration of the dusty phase for G1 be at least $13 \mathrm{yr}$.

As the majority of stars in the field and in dense stellar clusters like the nuclear star cluster exist as multiplecomponent systems (e.g., Sana \& Evans 2011; Duchêne \& Kraus 2013; Prodan et al. 2015), it is not unreasonable that many of these could merge in the Galactic center and form extended envelopes of gas and dust. The probability of finding two merger products must also be considered, including the likelihood of finding two on highly eccentric orbits. Let us assume that the G1 and G2 binary systems formed in the most recent star formation event. Based on estimates of merger timescales that include the eccentric Kozai mechanism, 
Stephan et al. (2016) estimated that $\lesssim 20 \%$ of all binaries formed would merge within the first few million years. Thus, if 1000 binaries with a comparable luminosity to G1 have formed from the $10^{4} M_{\odot}$ starburst event, then $\sim 200$ mergers will occur. We estimate that the G1 and G2 systems crossed their individual Roche limits and merged between $10^{4}-10^{6} \mathrm{yr}$ after the last star formation episode. The duration of the dusty, red phase of the merger depends on the mass of the progenitors and the relaxation timescale and is not well constrained; it may be as long as a few Myr (Stephan et al. 2016), and, after core merger, the resulting star takes a Kelvin-Helmholtz time (a few Myr) to contract to a compact star. Thus, a significant number of the mergers may still be visible as dusty, red objects depending critically on the duration of the dusty, red phase. The similarity of the orbital planes and eccentricities is also not unexpected in the merger hypothesis, given that G1 and G2 may have a common origin in the disk of young stars and that observational bias favors the recognition of fast-moving objects near the SMBH.

The present high eccentricities of G1 and G2 in their respective orbits around $\mathrm{Sgr} \mathrm{A}^{*}$ are consistent with binary systems that have been affected by the eccentric Kozai-Lidov mechanism (Kozai 1962; Lidov 1962; Naoz 2016), because binary systems perturbed by a massive third body are more likely to merge on highly eccentric outer orbits (Naoz \& Fabrycky 2014; Naoz 2016; Stephan et al. 2016). The detailed dynamics involved in the eccentric Kozai-Lidov mechanism that can yield merger products have been discussed in detail in the literature (see the review by $\mathrm{NaOz} 2016$ ). The merger rate and merger-induced products have been discussed in Prodan et al. (2015) and Stephan et al. (2016).

Merged binary systems undergo many physical changes as the merger occurs. For example, there is usually an optical outburst immediately following the physical merging of the stars, along with an evolution of the spectral type (e.g., Tylenda et al. 2011, Nicholls et al. 2013). The very few examples that have been published thus far have been inferred to be merged binaries because of optical periodic variability from the binary system before the outburst and the absence of any periodicity from the system following the outburst (e.g., Tylenda et al. 2011). Searches are underway for bursting or periodic sources in the Galactic center that may be mergers at different stages (A. Gautam et al. 2017, in preparation).

Several other hypotheses exist that could describe the observables of G1, such as edge-on, protoplanetary disks around young, low-mass stars (Murray-Clay \& Loeb 2012); disks around older stars (Miralda-Escudé 2012); or some other tidal disruption phenomenon involving a stellar object. Further observations, in particular of G2 after its periapsis transit, and more comprehensive statistics of infrared excess sources and intact binaries at the Galactic center will help to shed light on the nature of these objects.

\section{Conclusions}

G1 has several observable properties similar to those of the mysterious G2 object: it is a cold source in the Galactic center that has hydrogen recombination emission (at $\mathrm{Br} \gamma$ ) and has recently passed very close to Sgr $\mathrm{A}^{*}$. Our orbital fits indicate that G1 and G2 lie on similar orbital planes but have different arguments of periapsis, indicating that these objects are not part of the same gas streamer. In contrast to G2, G1 was originally well-resolved at $\mathrm{L}^{\prime}(3.8 \mu \mathrm{m})$ but became compact after periapsis. This additional information strongly supports the idea that there is a central, stellar object embedded in a gas- and dust-filled envelope.

We hypothesize that G1 may be a binary merger product due to the similarities to observed merger systems (see Section 5.2): notably, it has a large inferred size and high infrared luminosity. This would be a natural explanation for many unsolved questions regarding other populations in the Galactic center, including the young stars in the S-star cluster, which may have resulted from the mergers of binaries interacting with Sgr A* followed by relaxation back to the main sequence. G1 and G2 are also not the only objects with these observed properties in the Galactic center, as at least four others exist in close proximity to Sgr A* (Sitarski et al. 2014). Further studies of these additional sources will indicate whether all of the sources have common characteristics such as $\mathrm{Br} \gamma$ emission and whether they share a common origin or production mechanism.

Support for this work was provided by NSF grants AST0909218 and AST-1412615, the Levine-Leichtman Family Foundation, the Preston Family Graduate Fellowship (held by B.N.S. and A.B.), and the UCLA Graduate Division Dissertation Year Fellowship (held by B.N.S). The W. M. Keck Observatory is operated as a scientific partnership among the California Institute of Technology, the University of California, and the National Aeronautics and Space Administration. The authors wish to recognize that the summit of Maunakea has always held a very significant cultural role for the indigenous Hawaiian community. We are most fortunate to have the opportunity to observe from this mountain. The Observatory was made possible by the generous financial support of the W. M. Keck Foundation. B.N.S. also thanks James Larkin for his engaging discussions about the nature of G1 and G2, Alexander Stephan for his comments, and Ann-Marie Madigan for her thoughts on G1. We thank Arezu Dehghanfar for help with preparing the manuscript. We thank the anonymous referee for providing fruitful comments.

\section{Appendix A \\ $\mathbf{K}^{\prime}$-derived Distortion Solution on $\mathbf{L}^{\prime}$ Data}

As stated in Section 2, we resampled all data $\left(\mathrm{K}^{\prime}, \mathrm{L}^{\prime}\right.$, and $\mathrm{Ms}$ ) with the geometric optical distortion solution from Yelda et al. (2010). This distortion solution was derived with $\mathrm{K}^{\prime}$ data only, so we tested whether this distortion solution was inappropriately applied to the $\mathrm{L}^{\prime}$ and Ms data sets. We therefore took one of our epochs of data (we chose 2005.580) and transformed the $\mathrm{L}^{\prime}$ positions as detected by StarFinder (see Section 3.1.1) into the $\mathrm{K}^{\prime}$ coordinate system. We allowed for first-order translation, rotation, and pixel scale adjustments between the two frames that were independent in $x$ and $y$. The results from this alignment are shown in the left panel of Figure 15, where each arrow represents the difference in position for stars identified at both $\mathrm{K}^{\prime}$ and $\mathrm{L}^{\prime}$. As there is no noticeable rotation or structure indicated by the arrows, we conclude that applying the distortion correction for $\mathrm{L}^{\prime}$ data is therefore adequate. The right panel of Figure 15 shows a histogram of the difference between the $\mathrm{K}^{\prime}$ - and $\mathrm{L}^{\prime}$-transformed coordinates in both $x$ and $y$. The FWHMs of these histograms are less than the positional errors found in Table 2. 

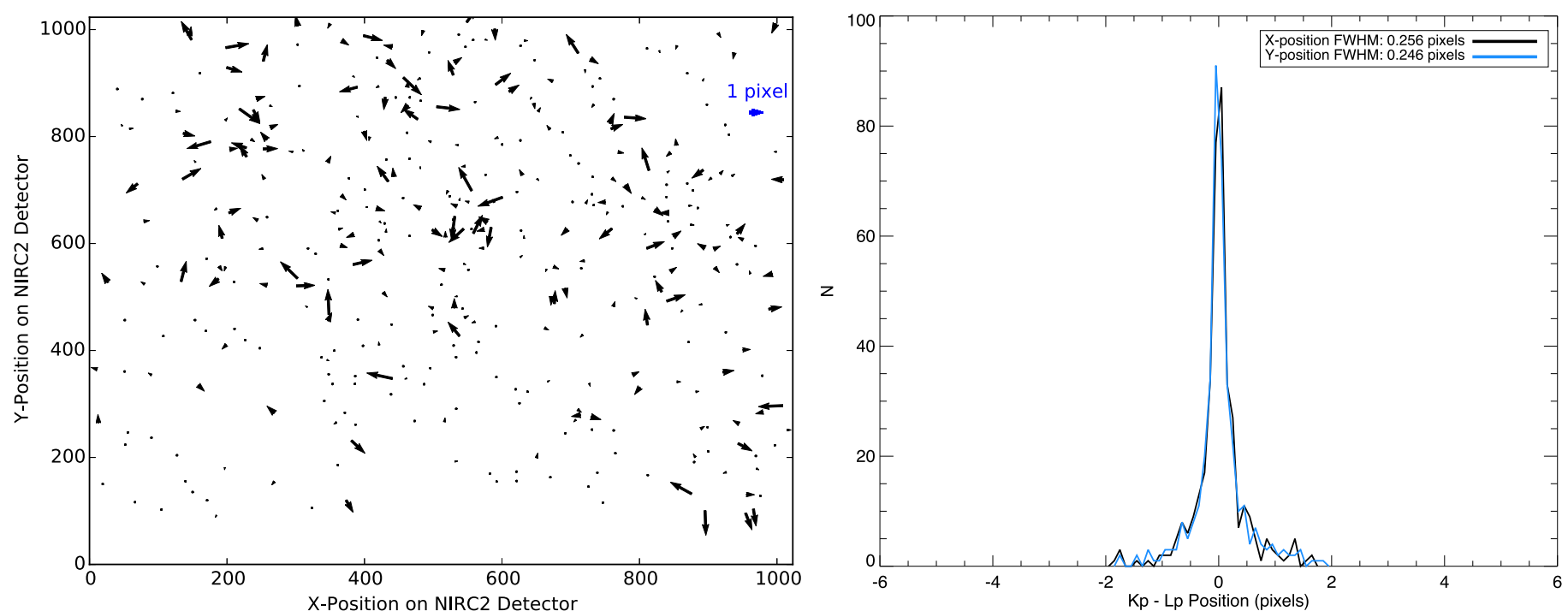

Figure 15. Left: difference between the $\mathrm{K}^{\prime}$ - and $\mathrm{L}^{\prime}$-transformed positions across the field of view of NIRC2. The arrows originate at the $\mathrm{K}^{\prime}$ position and end at the position of the $\mathrm{L}^{\prime}$-transformed-to- $\mathrm{K}^{\prime}$ coordinate system points. There is no preferential position or rotation of the arrows, so using the $\mathrm{K}^{\prime}$-data-derived distortion solution is completely adequate. Right: histogram of the difference between the $\mathrm{K}^{\prime}$ - and $\mathrm{L}^{\prime}$-transformed positions. The FWHMs of the distributions are less than the astrometric errors of the data.

Table 6

Jackknife Parameters

\begin{tabular}{|c|c|}
\hline Orbital Parameter & Jackknife Parameters \\
\hline $\begin{array}{l}X \text {-position of } \operatorname{Sgr~A}^{*}\left(x_{0}, \text { mas }\right) \\
Y \text {-position of } \operatorname{Sgr~A}^{*}\left(y_{0}, \text { mas }\right) \\
\Delta \text { R.A. velocity of } \operatorname{Sgr~A}^{*}\left(V_{x}, \operatorname{mas~yr}^{-1}\right) \\
\Delta \text { Decl. velocity of } \operatorname{Sgr}^{*}\left(V_{y}, \operatorname{mas~yr}^{-1}\right) \\
\text { Radial velocity of } \operatorname{Sgr} \mathrm{A}^{*}\left(V_{z}, \mathrm{~km} \mathrm{~s}^{-1}\right) \\
\text { Distance to } \operatorname{Sgr} \mathrm{A}^{*}\left(R_{0}, \mathrm{kpc}\right) \\
\text { Mass of } \operatorname{Sgr} \mathrm{A}^{*}\left(M, \text { millions of } M_{\odot}\right) \\
\end{array}$ & $\begin{aligned} 2.2 & \pm 0.3 \\
-4.3 & \pm 0.3 \\
0.11 & \pm 0.02 \\
0.67 & \pm 0.03 \\
-19.3 & \pm 3.7 \\
7.85 & \pm 0.06 \\
3.92 & \pm 0.06\end{aligned}$ \\
\hline \multicolumn{2}{|l|}{ G1 Parameters } \\
\hline $\begin{array}{l}\text { Periapsis passage distance }\left(a_{\min }, \mathrm{au}\right) \\
\text { Time of closest approach }\left(T_{0}, \mathrm{yr}\right) \\
\text { Eccentricity }(e) \\
\text { Argument of periapsis }(\omega, \mathrm{deg}) \\
\text { Inclination }(i, \mathrm{deg}) \\
\text { Position angle of the ascending node }(\Omega, \mathrm{deg})\end{array}$ & $\begin{aligned} 292 & \pm 44 \\
2001.3 & \pm 0.2 \\
0.993 & \pm 0.002 \\
117 & \pm 4 \\
109 & \pm 1 \\
86 & \pm 6\end{aligned}$ \\
\hline
\end{tabular}

\section{Appendix B}

\section{Jackknife-derived Estimates of the Variance of G1's Orbital Parameters and the Black Hole Parameters}

To determine whether our errors for the orbit of G1 and the black hole parameters capture at least part of the systematic errors due to potential outliers, we used a jackknife resampling technique to determine the variance of each of G1's orbital parameters while simultaneously fitting S0-2, S0-38, and G1. In each of our orbital fits, we dropped one epoch of observations and determined the jackknife variance over all orbital fits. Our recovered jackknife parameters are listed in Table 6 . The values and associated error bars calculated from this jackknife analysis are consistent with what is reported in Table 4.

\section{ORCID iDs}

G. Witzel (1) https://orcid.org/0000-0003-2618-797X

M. R. Morris (1) https://orcid.org/0000-0002-6753-2066

J. R. Lu (1) https://orcid.org/0000-0001-9611-0009
S. Naoz (1) https://orcid.org/0000-0002-9802-9279

A. Boehle (i) https://orcid.org/0000-0003-0439-7634

S. Yelda (1) https://orcid.org/0000-0001-5036-4329

\section{References}

Anninos, P., Fragile, P. C., Wilson, J., \& Murray, S. D. 2012, ApJ, 759, 132 Baganoff, F. K., Bautz, M. W., Brandt, W. N., et al. 2001, Natur, 413, 45 Ballone, A., Schartmann, M., Burkert, A., et al. 2013, ApJ, 776, 13 Bartko, H., Martins, F., Fritz, T. K., et al. 2009, ApJ, 697, 1741 Becklin, E. E., Zuckerman, B., McLean, I. S., \& Geballe, T. R. 1994, ApJ, 430, 774

Boehle, A., Ghez, A. M., Schödel, R., et al. 2016, ApJ, 830, 17 Buchholz, R. M., Schödel, R., \& Eckart, A. 2009, A\&A, 499, 483 Burkert, A., Schartmann, M., Alig, C., et al. 2012, ApJ, 750, 58 Clénet, Y., Rouan, D., Gendron, E., et al. 2004, A\&A, 417, L15 Clénet, Y., Rouan, D., Gratadour, D., et al. 2005, A\&A, 439, L9 Diolaiti, E., Bendinelli, O., Bonaccini, D., et al. 2000, A\&AS, 147, 335

Do, T., Ghez, A. M., Morris, M. R., et al. 2009a, ApJ, 703, 1323

Do, T., Ghez, A. M., Morris, M. R., et al. 2009b, ApJ, 691, 1021

Do, T., Martinez, G. D., Yelda, S., et al. 2013, ApJL, 779, L6

Draine, B. T. 2003, ARA\&A, 41, 241

Duchêne, G., \& Kraus, A. 2013, ARA\&A, 51, 269

Eckart, A., Mužić, K., Yazici, S., et al. 2013, A\&A, 551, A18

Genzel, R., Becklin, E. E., Wynn-Williams, C. G., et al. 1982, ApJ, 255, 527

Genzel, R., Schödel, R., Ott, T., et al. 2003, ApJ, 594, 812

Ghez, A. M., Duchêne, G., Matthews, K., et al. 2003, ApJL, 586, L127

Ghez, A. M., Hornstein, S. D., Lu, J. R., et al. 2005a, ApJ, 635, 1087

Ghez, A. M., Klein, B. L., Morris, M., \& Becklin, E. E. 1998, ApJ, 509, 678

Ghez, A. M., Morris, M., Becklin, E. E., Tanner, A., \& Kremenek, T. 2000 , Natur, 407,349

Ghez, A. M., Salim, S., Hornstein, S. D., et al. 2005b, ApJ, 620, 744

Ghez, A. M., Salim, S., Weinberg, N. N., et al. 2008, ApJ, 689, 1044 Ghez, A. M., Wright, S. A., Matthews, K., et al. 2004, ApJL, 601, L159

Gillessen, S., Eisenhauer, F., Trippe, S., et al. 2009, ApJ, 692, 1075

Gillessen, S., Genzel, R., Fritz, T. K., et al. 2012, Natur, 481, 51

Gillessen, S., Genzel, R., Fritz, T. K., et al. 2013a, ApJ, 763, 78

Gillessen, S., Genzel, R., Fritz, T. K., et al. 2013b, ApJ, 774, 44

Hornstein, S. D., Matthews, K., Ghez, A. M., et al. 2007, ApJ, 667, 900

Hurley, J. R., Pols, O. R., \& Tout, C. A. 2000, MNRAS, 315, 543

Kozai, Y. 1962, AJ, 67, 591

Larkin, J., Barczys, M., Krabbe, A., et al. 2006, Proc. SPIE, 6269, 62691A

Lau, R. M., Herter, T. L., Morris, M. R., Becklin, E. E., \& Adams, J. D. 2013, ApJ, 775, 37

Lau, R. M., Herter, T. L., Morris, M. R., Li, Z., \& Adams, J. D. 2015, Sci, 348, 413 Levin, Y., \& Beloborodov, A. M. 2003, ApJL, 590, L33

Lidov, M. L. 1962, P\&SS, 9, 719 
Lu, J. R., Ghez, A. M., Hornstein, S. D., et al. 2009, ApJ, 690, 1463

Madigan, A.-M., McCourt, M., \& O’Leary, R. 2017, MNRAS, 465, 2310

McCourt, M., \& Madigan, A.-M. 2016, MNRAS, 455, 2187

Meyer, L., Ghez, A. M., Schödel, R., et al. 2012, Sci, 338, 84

Meyer, L., Ghez, A. M., Witzel, G., et al. 2014, in IAU Proc. 303, The Galactic Center: Feeding and Feedback in a Normal Galactic Nucleus, ed. L. Sjouwerman, C. C. Lang, \& J. Ott (Cambridge: Cambridge Univ. Press), 264 Meyer, L., Witzel, G., Longstaff, F. A., \& Ghez, A. M. 2014, ApJ, 791, 24 Miralda-Escudé, J. 2012, ApJ, 756, 86

Morsony, B., Gracey, B., Workman, J., \& Yoon, D. 2015, arXiv:1508.00384 Murray-Clay, R. A., \& Loeb, A. 2012, NatCo, 3, 1049

Nakano, S., Nishiyama, K., Kabashima, F., et al. 2008, IAUC, 8972, 1

Naoz, S. 2016, ARA\&A, 54, 441

Naoz, S., \& Fabrycky, D. C. 2014, ApJ, 793, 137

Nicholls, C. P., Melis, C., Soszyński, I., et al. 2013, MNRAS, 431, L33

Paumard, T., Genzel, R., Martins, F., et al. 2006, ApJ, 643, 1011

Pfuhl, O., Gillessen, S., Eisenhauer, F., et al. 2015, ApJ, 798, 111

Phifer, K., Do, T., Meyer, L., et al. 2013, ApJL, 773, L13

Ponti, G., De Marco, B., Morris, M. R., et al. 2015, MNRAS, 454, 1525

Prodan, S., Antonini, F., \& Perets, H. B. 2015, ApJ, 799, 118

Rudy, R. J., Lynch, D. K., Russell, R. W., et al. 2008a, IAUC, 8976, 1

Rudy, R. J., Lynch, D. K., Russell, R. W., et al. 2008b, IAUC, 8997, 1

Sana, H., \& Evans, C. J. 2011, in IAU Symposium, Vol. 272, Active OB Stars: Structure, Evolution, Mass Loss, and Critical Limits, ed. C. Neiner et al. (Cambridge: Cambridge Univ. Press), 474
Schartmann, M., Burkert, A., Alig, C., et al. 2012, ApJ, 755, 155

Schödel, R., Morris, M. R., Muzic, K., et al. 2011, A\&A, 532, A83

Schödel, R., Najarro, F., Muzic, K., \& Eckart, A. 2010, A\&A, 511, A18

Scoville, N., \& Burkert, A. 2013, ApJ, 768, 108

Sitarski, B., Do, T., Witzel, G., et al. 2014, AAS Meeting, 223, 238.05

Sitarski, B., Ghez, A. M., Morris, M., et al. 2015, AAS Meeting, 225, 102.07

Stephan, A. P., Naoz, S., Ghez, A. M., et al. 2016, MNRAS, 460, 3494

Stolte, A., Morris, M. R., Ghez, A. M., et al. 2010, ApJ, 718, 810

Tielens, A. G. G. M., McKee, C. F., Seab, C. G., \& Hollenbach, D. J. 1994, ApJ, 431, 321

Tokunaga, A. T. 2000, in Allen's Astrophysical Quantities, ed. A. N. Cox (4th ed,; New York: Springer), 143

Tylenda, R., Hajduk, M., Kamiński, T., et al. 2011, A\&A, 528, A114

Tylenda, R., \& Kamiński, T. 2016, A\&A, 592, A134

Tylenda, R., Kamiński, T., Udalski, A., et al. 2013, A\&A, 555, A16

Valencia-S., M., Eckart, A., Zajaček, M., et al. 2015, ApJ, 800, 125

van Dam, M. A., Sasiela, R. J., Bouchez, A. H., et al. 2006, Proc. SPIE, 6272,627231

Witzel, G., Ghez, A. M., Morris, M. R., et al. 2014, ApJL, 796, L8

Wizinowich, P. L., Chin, J., Johansson, E., et al. 2006, Proc. SPIE, 6272, 6272709

Wynn-Williams, C. G., Becklin, E. E., Matthews, K., \& Neugebauer, G. 1978, MNRAS, 183, 237

Yelda, S., Ghez, A. M., Lu, J. R., et al. 2014, ApJ, 783, 131

Yelda, S., Lu, J. R., Ghez, A. M., et al. 2010, ApJ, 725, 331 